UCRL-JC-131422

PREPRINT

\title{
Searches for Invisible Axions
}

\author{
L. J. Rosenberg \\ K. A. van Bibber
}

This paper was prepared for submittal to

Physics Reports

July 1, 1998

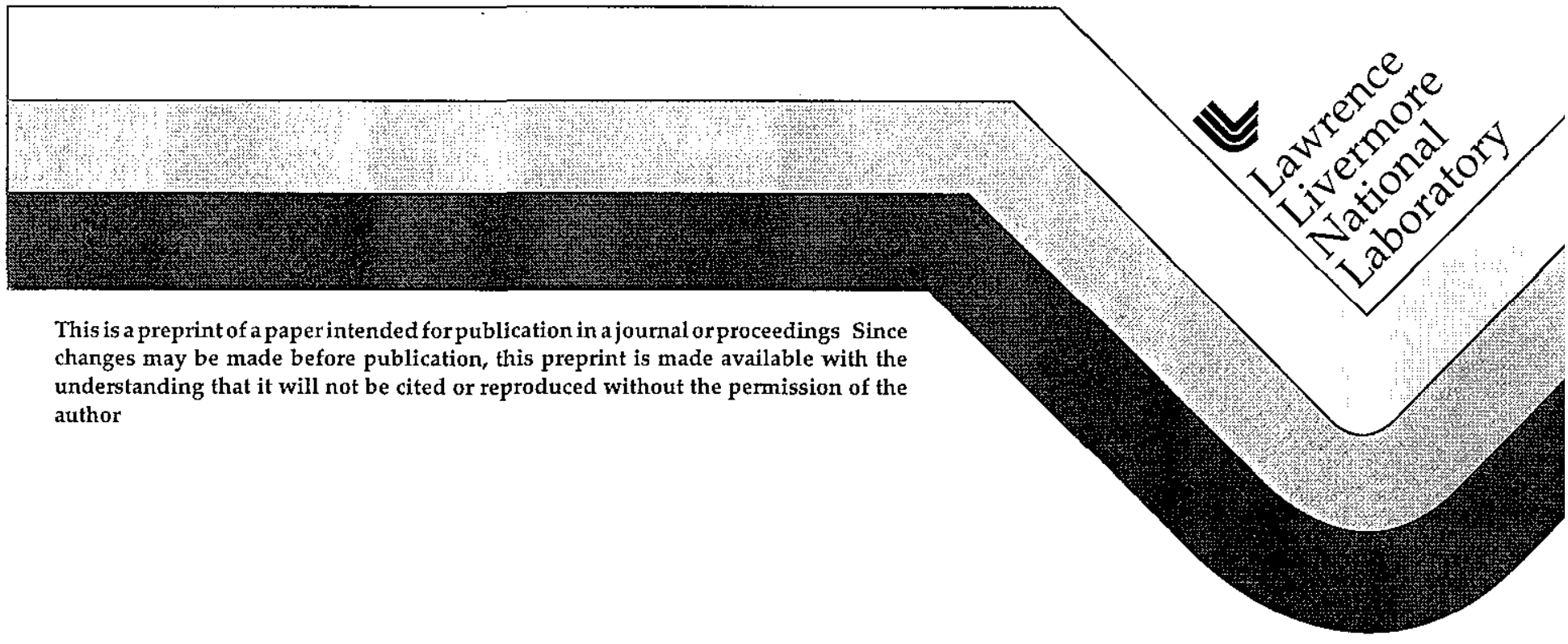




\section{DISCLAIMER}

This document was prepared as an account of work sponsored by an agency of the United States Government Neither the United States Government nor the University of California nor any of their employees, makes any warranty, express or implied, or assumes any legal liability or responsibility for the accuracy, completeness, or usefulness of any information, apparatus, product, or process disclosed, or represents that its use would not infringe privately owned rights Reference herein to any specific commercial product, process, or service by trade name, trademark, manufacturer, or otherwise, does not necessarily constitute or imply its endorsement, recommendation, or favoring by the United States Government or the University of California The views and opinions of authors expressed herein do not necessarily state or reflect those of the United States Government or the University of California, and shall not be used for advertising or product endorsement purposes 


\title{
Searches for Invisible Axıons
}

\author{
Leslie J Rosenberg \\ Department of Physics and Laboratory for Nuclear Science \\ Massachusetts Institute of Technology \\ 77 Massachusetts Ave., Cambridge, MA 02139, USA
}

Karl A. van Bibber

Physics \& Space Technology Directorate

Lawrence Livermore National Laboratory

7000 East Ave., Livermore, CA 94550, USA

\begin{abstract}
The axion, a hypothetical elementary particle, emerged from a compelling solution to the Strong-CP problem in QCD. Subsequently, the axion was recognized to be a good Cold Dark Matter candidate. Although dark matter axions have only feeble couplings to matter and radiation, extremely sensitive searches are underway around the world. We review the state of experiments searching for the axion through its coupling to photons. Besides answering an outstanding question in particle physics, the discovery of dark matter axions may provide a detailed history of the formation of our Milky Way galaxy through the structure of the axion signal.
\end{abstract}

We dedicate this review to the memory of Professor David N Schramm, who commissioned this work but whose untimely death prevented him from seeing its completion $\mathrm{He}$ was an extraordinary figure in modern astrophysics and cosmology; his loss to the field is incalculable. Most importantly, he will be remembered as a wonderful human being, a great friend and a model to younger scientists. 


\section{Contents}

1 Introduction 3

11 Axions in Particle Physics 4

12 Constraints on the Axion Mass and Couplings 6

2 The Search for Axionic Dark Matter 11

21 Axions as Halo Dark Matter 11

22 The Cavity Microwave Experiment 12

23 First-Generation Experiments 13

24 Second-Generation Experiments 15

3 Other Methods for $m_{A}<\mathcal{O}(1 \mathrm{eV})$

31 Axion-Photon Mixing 22

32 Purely Laboratory Experiments through the Axion-Photon Coupling 23

33 Cosmological/Astrophysical axions 26

34 New Macroscopic Forces 28

4 Summary and Outlook 29

$\begin{array}{ll}\text { References } & 31\end{array}$ 



\section{Introduction}

Peccei-Quinn (PQ) symmetry, from which the axion arises, still stands after two decades as the most minimal and elegant extension to the Standard Model to enforce Strong-CP in particle physics.[1-3] Within its presently allowed mass range $\left(10^{-(6-2)} \mathrm{eV}\right)$, it is also a good particle dark matter candidate While the couplings of such light axions to matter and radiation would be exceedingly weak, it is realistic to expect that the status of the axion may be definitively resolved within the next decade.

The original axion with the $\mathrm{PQ}$ symmetry-breaking scale, $f_{A} \sim f_{E W} \sim$ $250 \mathrm{GeV}$ implied an axion mass of a few hundred $\mathrm{keV}$, and couplings with matter and radiation that would have made it readily observable in acceleratorand reactor-based experiments [4] It was quickly ruled out, and axion models were constrùcted with $f_{A}>>f_{E W}$, rendering the mass and all couplings extremely small, thus giving rise to the name the 'invisible axion'. In 1983, Pierre Sikivie of the University of Florida showed how halo dark matter axions could be detected by their Primakoff conversion to microwave photons in a high- $Q$ resonator permeated by an intense magnetic field.[5] First-generation experiments at BNL[6] and the University of Florida[7] demonstrated the experimental technique, and laid the groundwork for second-generation efforts in the US [8] and Japan [9] of which the former has finally reached sensitivity to realistic axion models with best-fit local halo Cold Dark Matter (CDM) densities. Upgrades are being planned to further increase sensitivity and search rate, to maximize the chances of the discovery of the axion.

While the scope of this review principally covers the searches for axions constituting our own galactic dark matter, we have also included purely laboratory experiments applicable to light pseudoscalars in the $m_{A}<1 \mathrm{eV}$ range, and searches for axions from astrophysical sources, based on axion-photon mixing While these latter are attractive in virtue of being broad-band in mass and independent of assumptions concerning the axionic content of our halo, they fall far short of the sensitivity required to detect standard axions, and it is not obvious how they could be improved significantly. Accelerator searches which ruled out the original axion, and 'fifth-force' experiments are discussed only briefly The theoretical motivation for the axion, the presently understood constraints, and the limits specifically derived from stellar evolution will only be treated in a summary way in the introduction, as these three topics have been reviewed in detail in this journal by Kim,[4] Turner[10] and Raffelt. [11] 


\section{Axions in Particle Physics}

\section{The $\theta$ Problem in $Q C D$}

Quantum Chromo Dymanics (QCD), the theory of the strong interactions, has proven remarkably successful Its one loose end, however is the StrongCP problem The non-Abelian nature of QCD should introduce T, P and CP violating effects, and in particular there should be a substantial $\mathrm{CP}$ violating neutron electric dipole moment $(\mathrm{edm})$. The vanishingly small neutron edm is therefore a genuine mystery

The source of CP violating interactions in QCD is traced to the complexity of the QCD vacuum The QCD vacuum has gluon fields in their lowest energy configuration, and there are many degenerate vacua The various vacua can be classified by winding number $n$-the non-Abelian nature allows non-zero $n$-and gauge transformations can change one winding number vacuum into another The physical vacuum of QCD is constructed by a gauge invariant Bloch-wave-like superposition of vacua:

$$
|\Theta\rangle=\sum_{n} e^{-i n \Theta}|n\rangle
$$

Effects of the $\Theta$ vacuum on vacuum transition amplitudes can be subsumed in a new effective non-perturbative term in the QCD Lagrangian proportional to $\bar{\Theta} G \tilde{G}$, with $G$ and $\tilde{G}$ the gluon field strength tensor and its dual, and $\bar{\Theta}=\Theta+\arg \operatorname{det} M$, where $M$ is the quark mass matrix The parameter $\bar{\Theta}$ takes contributions from the QCD vacuum $\Theta$ and phases from the quark mass matrix. The $G \tilde{G}$ term in the Lagrangian is a total derivative and does not contribute to classical equations of motion or perturbative effects However, the term is explicitly $\mathrm{CP}$ violating and can induce non-perturbative $\mathrm{CP}$ violating effects With a $\bar{\Theta}$ of order 1, the neutron can be shown[12] to have an expected electric dipole moment of order $10^{-15} \mathrm{e} \mathrm{cm}$. Current limits are $\sim 10^{-25} \mathrm{ecm},[13]$ and these limits in the context of the $\Theta$ vacuum restricts the magnitude of $\bar{\Theta}$ to less than a few times $10^{-10}$ A comprehensive discussion of the role of $\Theta$ is given by Cheng [14]

\section{Axions and Strong-CP}

That $\bar{\Theta}$ has contributions from both QCD (through the $\Theta$ vacuum) and weak interactions (through the quark mass matrix) makes the Strong-CP problem a far more serious problem More than just finding why one parameter should be zero, we need to explain the near-perfect cancellation of two independent but finite effects Among the ideas for evading this problem,[15] we find most compelling the one invoking the axion 
The axion resulted from the solution proposed by Peccei and Quinn [1] They showed that a minimal extension of the Higgs sector endows the Standard Model with a global U(1) symmetry, the Peccei-Quinn (PQ) symmetry, to be broken at some scale $f_{P Q}$. A new effective term arises in the QCD Lagrangian proportional to $\left(\phi_{A} / f_{P Q}\right) \bar{\Theta} G \tilde{G}$, with $\phi_{A}$ the axion field, and constant of proportionality dependent on the value of the axion color anomaly The sum of $\Theta$ and anomaly terms, taken as a classical potential, is minimized at some axion vacuum expectation value proportional to $\bar{\Theta} f_{P Q}$ At this value of the axion field, the $\mathrm{CP}$ violating $G \tilde{G}$ terms, including those giving rise to a neutron electric dipole moment, almost completely vanish Weinberg and Wilczek subsequently pointed out that since a continuous symmetry is broken, there must also be an associated Goldstone boson-the axion [2,3] Although the axion starts out as a massless Goldstone boson, it eventually acquires an effective mass (as does, e g., the $\eta$ ) through intermediate states coupled to its color axial anomaly The theory of the axion and various models incorporating $P Q$ symmetry is thoroughly discussed by Kim [4]

\section{Mass and Couplings}

The axion mass and the PQ symmetry-breaking scale $f_{P Q}$ are related through

$$
m_{A}=\frac{\sqrt{z}}{1+z} \frac{f_{\pi} m_{\pi}}{f_{P Q} / N}
$$

with $z$ is the ratio of $\mathrm{u}$ - and d-quark masses (presumably $\sim 0.5$ ), and an integer $N$ the axion color anomaly. For the purposes of this review, the model dependence will be treated phenomenologically, entering through $N$, and the axion electromagnetic anomaly $E$ Taking an experimental point of view, we avoid detailing various scenarios from which $P Q$ symmetry could originate, and concentrate first on whether PQ symmetry is a valid description of nature at all

The strength of the axion's couplings to normal matter and radiation are given by effective coupling constants $g_{A \gamma \gamma}, g_{A e e}, g_{A p p}$, etc., for the axion coupling to photons, electrons and protons. Since the elementary axion couplings are model dependent, these effective couplings are model dependent as well For instance, the effective axion-two-photon coupling constant is

$$
g_{A \gamma \gamma}=\frac{\alpha / 2 \pi}{f_{P Q} / N}(E / N-2(4+z) / 3(1+z)),
$$

where the factor $2(4+z) / 3(1+z)$ containing ratios of light quark masses is approximately 195 The tree level coupling of axions to color is fundamental to the axion's role in solving the strong $\mathrm{CP}$ problem The tree level coupling of the axions to charged leptons however is optional, here, different theories 
allow different couplings. Extremes of lepton couplings are cases with no tree level axion coupling to electrons (called 'hadronic axions', one implementation being the KSVZ axion[16]) and axions where tree level quark and electron couplings are of the same strength (an example being axions motivated by a simple GUTs scenario, called the DFSZ axion[17])

Generically, all effective coupling constants of axions with normal matter and radiation depend on the inverse of the symmetry-breaking scale $f_{P Q}$, and are therefore linear in the axion mass Extremely light axions thus possess extremely weak couplings, and for that reason were termed 'invisible axions' The most sensitive of the current round of axion searches look for these invisible axions through their coupling with two photons While there is nothing to forbid $E / N$ from having the unfortunate value $\sim 195$ which effectively suppresses the axion-photon coupling, in the simple DFSZ GUTs model for example, $E / N=8 / 3$, and in the KSVZ model $E / N=0$

\section{Constraints on the Axion Mass and Couplings}

\section{Reactor and Accelerator Searches}

Early searches for axions took as a starting point that the PQ symmetry-breaking scale be near the weak scale. The resultant axions are relatively strongly coupled to normal matter and radiation and would have been produced and detected in reactor and accelerator experiments. That no such axions were found requires the $P Q$ scale be considerably greater than the weak scale. Following we give an updated summary of these early searches built on the previous reviews Refs. [4] and [14]

\section{Reactor Experiments}

Fission reactions in nuclear reactors are a copious source of $\bar{\nu}_{e}$. Very roughly, each $\bar{\nu}_{\mathrm{e}}$ is accompanied by a prompt $\gamma$; this mostly from $\mathrm{E} 1$ transitions, but with also a small contribution from M1 transitions [18] Most of the M1 $\gamma$ 's come from isovector transitions. Should there be an axion, there would be a pseudoscalar contribution to the M1 rate via axion emission These axions would escape the reactor, decay into two photons, and be detected by conventional scintillator detectors. Alternatively, the axion could scatter into a photon from the Compton-like process $A+e^{-} \rightarrow \gamma+e^{-}$, with the resultant $\gamma$ again detected One group[19] placed $\mathrm{NaI}$ detectors approximately $1 \mathrm{~m}$ from the Savannah River reactor core and counted photons depositing energy greater than $15 \mathrm{MeV}$ The found a corrected rate of $-160 \pm 260$ events/day, while a weak-scale axion would have given more than 1000 counts

An earlier experiment[20] looked for the effect of weak neutral current neu- 
trino-induced disintegration of deuterium $\bar{\nu}_{e}+d \rightarrow p+n+\bar{\nu}_{e}$ The corrected disintegration rate was $-3 \pm 7$ per day An axion with energy greater than $2.2 \mathrm{MeV}$ would have induced disintegration via nucleon scattering. Such axioninduced disintegrations would have contributed at least 1000 counts per day to the corrected disintegration rate

\section{Nuclear Deexcitations}

An excited nucleus can deexcite via emission of an axion, with the axion carrying away spin-parity $J^{P}=0^{-}, 1^{+}, 2^{-} \quad$ The contribution to isoscalar and isovector transitions from an axion carrying $J^{P}=1^{+}$has been calculated[18] and compared to upper limits to decay rates in excited ${ }^{137} \mathrm{Ba},{ }^{7} \mathrm{Li}$, and ${ }^{97} \mathrm{Nb}$ systems The measured upper limits on these rates are orders of magnitude less than the expected contribution from a weak-scale axion

\section{Beam Dumps}

In beam dump searches, axions would be produced in a target, penetrate through an earth shield, then interact with a detector. Early SLAC experiments[21] directed electrons from the linac onto a target Axions produced in the target from direct axion bremsstrahlung off the incident electrons would penetrate into a $55 \mathrm{~m}$ earth shield and then decay into muon pairs No such muon pairs were detected. Several groups at CERN (BEBC,[22] Gargamelle,[23] and CDHS[24]) looked for decay products of axions. Here, $400 \mathrm{GeV}$ protons from the SPS were directed on a target. Axions produced from nuclear bremsstrahlung would penetrate through about $1 \mathrm{~km}$ of earth shield between the target and detectors. The axion signature is excess lepton pairs. Weakscale axions would have resulted in perhaps hundreds of events, while no axion decays were detected above the very small neutral-current background.

Later SLAC beam dump experiments were motivated by a possible $1.8 \mathrm{MeV}$ mass axion source of $e^{+} e^{-}$coincidences seen in heavy ion collisions at the Gesellschaft für Schwerionforschung.[25] SLAC experiment E-141[26] stopped $9 \mathrm{GeV}$ electrons in 10 and $12 \mathrm{~cm}$ tungsten beam stops Axions radiated from electrons emerge from the back of the beam stop, decay, and decay products enter the SLAC $8 \mathrm{GeV}$ spectrometer for analysis. Hadronic and muon backgrounds were removed by a hydrogen Čerenkov counter and lead glass colorimeter. The onia decay experiments already excluded a weak-scale axion of this mass; E-141 excluded an unusual axion with preferential decays into $e^{+} e^{-}$pairs with appropriate lifetime to decay between the exit of the beam stop and the spectrometer SLAC experiment E-137[27] directed 30 Couloumbs of $20 \mathrm{GeV}$ electrons onto an aluminum and water beam dump. Downstream of the absorber was a hill of $179 \mathrm{~m}$ thickness, followed by the E-137 apparatus consisting mainly of an 8 radiation length shower calorimeter This experiment was sensitive to weak-scale axion decaying into $e^{+} e^{-}$of photon pairs 
with mass greater than a few $100 \mathrm{keV}$, as shown in Fig 1.

\section{Onia Decays}

The $1^{-}$heavy quark onia states $\mathrm{J} / \Psi$ and $\Upsilon$ can decay into $A+\gamma$ If the resultant axion lives long enough, the axion signature is a single photon, an almost background-free topology in onia decays Alternatively, for short-lived axions, axion decay products enter the detector The CUSB[28] and CLEO[29] collaborations at CESR, the Crystal Ball[30] group at SPEAR, and the LENA[31] collaboration at DORIS looked for the single photon event topology and at high confidence excluded a weak-scale axion A later CUSB study[32] ruled out shorter-lived weak-scale axions with event topology a single photon accompanied by two electromagnetic (e or $\gamma$ ) partners

\subsection{Stellar Evolution and Supernovae}

Powerful limits on exotic particles can be set by their influence on stellar evolution [33] The present upper limit on the axion mass by stellar evolution arguments is orders of magnitude better than that from accelerator- and reactor-based experiments The basis for the method is the fact that the evolution of any star is throttled by the rate at which thermal energy can be dissipated Stars are usually completely opaque to radiation produced in their interior, so stellar cooling is determined by radiation from a photosphere near the surface (In the case of the SN1987a, the same argument applies to neutrino radiation ) On the other hand, a very weakly-coupled exotic particle, even if only rarely produced, can efficiently transport energy directly out of the stellar interior In some cases, this 'cooling' mechanism accelerates the star's evolution and increases its temperature, as gravitational potential energy is converted into heat counteracting the loss of radiation pressure Limits are derived when clear discrepancies arise between stellar evolution models and observables We summarize below the most important stellar evolution limits for the axion.

At the center of the sun, about $10\left(m_{A} / \mathrm{eV}\right)^{2} \mathrm{erg} \mathrm{g}^{-1} \mathrm{sec}^{-1}$ DFSZ axions of mass $m_{A}$ would be created through Compton processes like $e+\gamma \rightarrow e+A$, to be compared with the nuclear energy release of a few $\mathrm{erg} \mathrm{g}^{-1} \mathrm{sec}^{-1}$ Based on our understanding of solar dynamics and the Sun's measured ${ }^{4} \mathrm{He}$ content and luminosity, it is unlikely that axions are at this moment removing more than about half of the nuclear energy, thereby constraining the DFSZ axion mass to less than about $1 \mathrm{eV}$ [34] Likewise, should there be an axion-induced temperature rise, the observed solar neutrino event rate would increase If anything, there is evidence for a "solar neutrino deficit" Taken together, solar evolution excludes DFSZ axion masses between $\sim 1 \mathrm{eV}$ and the solar central temperature of a few $\mathrm{keV}$ 
Main sequence stars will accumulate a helium core from hydrogen burning, and if sufficiently massive, the helium core will ignite The resulting red giant has a large diameter, but low surface temperature and luminosity. It exhausts its helium fuel in a very short time ( $100 \mathrm{Myr})$ and continues evolving into a compact object. Hadronic axions would be produced in red giants by a Primakoff process, $i e$ the fusion of a real photon with a virtual photon from a charged particle, $\gamma+Z e \rightarrow A+Z e$ The effect axion production would be to increase the helium burning rate and reduce the time a star spends as a red giant The fraction of red giants in a stellar population thereby declines, and statistical analyses of well-understood clusters result in an upper limit to the hadronic axion mass of around a few $\mathrm{eV}[35,33]$

Red giants set a much stronger limit for DFSZ axions, where axio-Compton production dominates, $\gamma+e \rightarrow A+e$. The helium core, supported only by electron degeneracy pressure, shrinks in size as it builds up, liberating gravitational binding energy and raising its temperature. Without axions, core cooling is throttled by neutrino radiation The axion production rate is inversely proportional to its mass, and like neutrino production, is proportional to a high power of the core temperature Assuming axions dominate energy transport, the core temperature then varies as an inverse power of the axion mass. A sufficiently massive DFSZ axion inhibits helium ignition entirely Detailed modeling excludes DFSZ axions with mass greater than $\sim 10^{-2} \mathrm{eV} \cdot[36,33]$ Of course, axions with mass much greater than the red giant core temperature near $10 \mathrm{keV}$ cannot be excluded by this argument.

The supernova SN1987A released $\sim 10^{53}$ ergs of energy, virtually all of it in neutrinos with a characteristic temperature of $\sim 10 \mathrm{MeV}$. The Kamiokande and IMB detectors together recorded 19 neutrinos spread over about 10 seconds, a result consistent with our understanding of supernovae dynamics and the number of light neutrino flavors. Early analyses demonstrated that axion radiation by nucleon-nucleon bremsstrahlung $N N \rightarrow N N A$ would have noticeably foreshortened the neutrino pulse for axion masses between $10^{-3} \mathrm{eV}$, and around $2 \mathrm{eV}$ This upper limit represents where nucleon-pair absorption would have suppressed free-streaming of axions out of the proto-neutron star.[37-39] More detailed treatments show the importance of nuclear mean-field effects in axion production These are difficult to quantify, but are unlikely to raise the lower limit to more than $10^{-2} \mathrm{eV}$ [33]

\section{Cosmological Production}

Laboratory experiments and astrophysics establish upper bounds to the axion mass In principle, cosmology imposes a lower bound, from the requirement that axions not overclose the Universe. One cannot be too specific, as we do not know which production mechanism would be predominant 


\section{Vacuum misalignment}

In the PQ picture, non-perturbative QCD effects establish a potential which drives the classical $\bar{\Theta}$ parameter to almost exactly zero, independent of its original value after symmetry-breaking. This happens in the low-temperature limit of the theory, $T \sim \Lambda_{Q C D}<<f_{A}$. However, the total energy in the axion field depends on the original misalignment angle $\bar{\theta}$, an unprescribed value between $-\pi$ and $\pi$ [40] A simple model is a pendulum at some random angle initially in a field-free environment, and then 'turning on' gravity [41] The axion field would have been a Bose condensate of zero temperature, and the present density of axions produced by the misalignment mechanism is given by[42]

$$
\Omega_{A}=085 \times 10^{ \pm 04}\left(\Lambda_{Q C D} / 200 \mathrm{MeV}\right)^{-07}\left(m_{A} / 10^{-5} \mathrm{eV}\right)^{-118} / h^{2},
$$

The Hubble factor $h=H / 100$ enters through expansion-driven damping, and the QCD scale $\Lambda_{Q C D}$ enters as the temperature where mass appears. The value of $\Omega_{A}$ presumes an effective $\bar{\Theta}=\pi / \sqrt{3}$, the r.m s value of the interval $-\pi$ to $\pi$. Physically this corresponds to the case where inflation either does not occur, or occurs before $\mathrm{PQ}$ symmetry-breaking, making the effective misalignment angle a composite value for a great number of causally disconnected volumes If inflation happens after PQ symmetry-breaking, a similar expression for $\Omega_{A}$ results, but proportional to $\bar{\Theta}^{2}$, a random number. In this case $\bar{\Theta}$ is the value pertaining to the entire visible Universe, which came from one minuscule preinflation volume While in this latter case, a value of $\bar{\Theta} \sim 0$ cannot be excluded, it should be presumed to be of order unity Under either scenario a nominal lower bound for the axion mass is $\sim 10^{-5} \mathrm{eV}$

\section{Axionic strings}

The axion is the Goldstone boson of a spontaneously broken global U(1) symmetry, which has strings as solutions to the equation of motion [43] These strings are nearly one-dimensional objects, either closed loops or infinitely long Assuming either that inflation never occurred, or occurred before the breaking of PQ symmetry, a network of strings develop. These interact and form loops, which then evaporate via axion radiation.[44] There still remains a sharp divergence between two schools concerning the relative importance of string radiation and vacuum misalignment mechanisms to the relic axion density [45] This is no business for an experimental review-suffice to say that the significantly higher axion mass limit found in detailed string simulations by one group can, at the outside of their uncertainties, be pushed down almost to the limit of the other, which incidentally coincides with the vacuum misalignment value

Figure 2 summarizes the present limits on the axion mass from stellar limits and cosmology [33] 


\section{The Search for Axionic Dark Matter}

\section{Axions as Halo Dark Matter}

\section{Spectral Shape of the Virialized Component}

In designing an experiment to look for dark matter axions, one needs to have an approximate idea of what one is looking for As will be seen below, the microwave cavity experiment measures the full energy of an axion, $i e$. the sum of its rest mass plus kinetic energy The halo axions looked for are gravitationally bound in our Milky Way galaxy, with a virial velocity of $\bar{\beta} \sim 10^{-3}$ Naively then, the axion energy distribution would be expected to be monochromatic to 1 part in $10^{6}$

To be more precise, the assumption is usually made that the halo axions occupy a spherical isothermal phase-space distribution. There is no evidence that such a CDM halo could not be rotating with respect to the galaxy, or that it might not be oblate or even triaxial. But proceeding under this assumption, the axion distribution would be a Maxwellian.

$$
f d v=4 \pi\left(\frac{\beta}{\pi}\right)^{3 / 2} v^{2} \exp \left(-\beta v^{2}\right) d v
$$

Based on the dynamics of our galaxy, the r.m.s velocity of our halo is $\bar{v}=\left\langle v^{2}\right\rangle^{1 / 2} \sim 270 \mathrm{~km} \mathrm{sec}^{-1}$, implying $\beta=(3 / 2) \bar{v}^{-2} \sim 220\left(\mathrm{~km} \mathrm{sec}^{-1}\right)^{-2}$ However, the laboratory "swims" through this distribution with a time-varying velocity $\vec{v}_{E}=\vec{v}_{S}+\vec{v}_{O}+\vec{v}_{R}$, the terms on the right representing the Sun's velocity in the galactic rest frame (magnitude $\sim 230 \mathrm{~km} \mathrm{sec}^{-1}$ ), the Earth's orbital velocity around the Sun (magnitude $\sim 29.8 \mathrm{~km} \mathrm{sec}^{-1}$ ), and the Earth's rotational velocity (magnitude at the equator $\sim 0465 \mathrm{~km} \mathrm{sec}^{-1}$ ). The observed axion velocity is then $\vec{v}_{A}=\vec{v}-\vec{v}_{E}$. The effect of this motion is to broaden the distribution, as well as giving rise to shape variations which are periodic both in one sidereal day, and one sidereal year:[46]

$$
\begin{array}{r}
f d E=2\left(\frac{\beta}{\pi}\right)^{1 / 2} \frac{d E}{m_{A} v_{E}} \exp \left(-\beta v_{E}^{2}-2 \beta E / m_{A}\right) \\
\times \sinh \left[2 \beta\left(2 E / m_{A}\right)^{1 / 2} v_{E}\right]
\end{array}
$$

The expected peak is still very narrow Expressed as the mean kinetic energy relative to the rest mass, the broadening is $\bar{v}_{A}^{2} / 2 c^{2} \sim 7 \times 10^{-7}$, or as the dispersion about the mean, $\widetilde{v}_{d i s}^{2} / 2 c^{2} \sim 53 \times 10^{-7}$. These results are shown in Figure 3. The relative sinusoidal variations on the axion kinetic energy 
(dispersion) are 55 (3 9)\% for the Earth's orbital motion, and $0093(0066) \%$ for the Earth's rotation

\section{Phase Space and Ultra-Fine Structure}

In the simple structure formation picture, once a density perturbation begins to grow, there is a stagnation radius within which CDM falls into the gravitational potential, and beyond which CDM continues to Hubble-expand outwards However as the structure grows and its stagnation radius increases, new CDM crosses within the radius and falls into the potential. As pointed out by Sikivie and coworkers, $[47,48]$ in the absence of dissipation the CDM phase space distribution in the halo should result in a spectrum of discrete lines in velocity (and energy) as measured at any point within, see Figure 4 The lowest energy lines are those which fell into the potential early on when the potential was shallowest, and have oscillated $\sim \mathcal{O}(100)$ times across the halo. The highest energy lines are those that have just fallen in, when the potential was the deepest, and are just crossing our Solar System for the first time, or have oscillated only a few times through the halo Extensive calculations have been done both with and without initial angular momentum, but the general features are the same These authors identify mechanisms that will eventually lead to a thermalization of the spectrum ( $\mathrm{g}$ scattering from globular clusters) and provide numerical estimates. But it seems very plausible that at least the few highest energy lines, each containing several percent of the .total axion signal should persist Taken seriously, there are two important implications for the microwave cavity experiment. First, as the primordial axion spectrum is truly a zero-temperature condensate $\left(\Delta E / E \sim 10^{-18}\right)$, the width of the line may be exceedingly narrow. Even $10^{-2}$ of the axion spectrum in one such line would lead a much higher $S / N$ than for the virialized spectrum, and thus a greatly increased discovery potential for the experiment if one can search for narrow lines Second, if the axion were found, the spectrum of such lines would provide a time-ordered history of our galactic formation

\subsection{The Cavity Microwave Experment}

Halo axions could be seen through their resonant conversion into photons in a high-Q cavity threaded by a magnetic field In practice, a tunable heliumcooled high- $Q$ cavity is placed in the bore of a superconducting solenoid, and the resonant frequency of its lowest TM mode is slowly swept while the cavity output is monitored for excess power from resonant axion conversions [5] The excess power is 


$$
\begin{aligned}
& P=96 \times 10^{-27} \text { Watt }\left(\frac{V}{1 \mathrm{~m}^{3}}\right)\left(\frac{B_{0}}{10 \text { Tesla }}\right)^{2} \\
& \times C\left(\frac{g_{\gamma}}{0.36}\right)^{2}\left(\frac{\rho_{\mathrm{A}}}{05 \times 10^{24} \mathrm{~g} / \mathrm{cm}^{3}}\right)\left(\frac{m_{\mathrm{A}}}{10 \mu \mathrm{eV}}\right) \min \left(Q_{\mathrm{L}}, Q_{\mathrm{A}}\right)
\end{aligned}
$$

with $V$ the volume of the cavity, $B_{0}$ the magnetic field strength, $C$ a modedependent form factor of order unity, $\rho_{A}$ the density of galactic halo axions at the Earth, $m_{A}$ the axion mass, $Q_{L}$ the loaded $Q$ of the cavity and $Q_{A} \sim 10^{6}$ the "quality factor" of the galactic halo axions, $i e$ the ratio of their total energy to their energy spread near Earth (The loaded $Q$ of the cavity incorporates both its ohmic losses $Q_{W}$ (wall) and the coupling of power out of the cavity $Q_{O}$ (out), $Q_{L}^{-1}=Q_{W}{ }^{-1}+Q_{O}^{-1}$ ) The signal-to-noise at resonance can be shown to be maximized when $Q_{L}=(1 / 3) Q_{W}$. Finally, $g_{\gamma} \sim(E / N-195) / 2$ denotes the axion-photon coupling A value $g_{\gamma} \sim 036$ is predicted for DFSZ axions, and -097 for KSVZ hadronic axions. One should appreciate how minuscule the expected signal is. With the nominal value of constants in the above expression, and a cavity of $Q_{L}=10^{5}$ at $1 \mathrm{~K}$ temperature, the blackbody power within the bandwidth of the axion peak is 30 times greater than the signal.

Unfortunately, the axion mass is unknown, as is the corresponding resonant frequency $\nu=m_{A} c^{2} / h$. As mentioned previously however, critical density in axions from the misalignment mechanism most likely correspond to an axion mass in the $1-10 \mu \mathrm{eV}$ range. This is what makes the first decade of the axionsearch window so promising. (As the cavity radius for the $\mathrm{TM}_{010}$ mode of interest is $R[\mathrm{~m}]=0115 / \nu[\mathrm{GHz}]$, and $1 \mathrm{GHz}=4.136 \mu \mathrm{eV}$, this mass range also corresponds to a convenient range of cavity diameters, $5-50 \mathrm{~cm}$.) The search rate for a constant signal to noise ratio $(s / n)$ is

$$
\begin{aligned}
& \frac{d \nu}{d t}=\frac{28 \mathrm{GHz}}{\text { year }}\left(\frac{4}{\mathrm{~s} / \mathrm{n}}\right)^{2}\left(\frac{V}{1 \mathrm{~m}^{3}}\right)^{2}\left(\frac{B_{0}}{10 \text { Tesla }}\right)^{4} \\
& \times C^{2}\left(\frac{g_{\gamma}}{0.36}\right)^{4}\left(\frac{\rho_{\mathrm{A}}}{05 \times 10^{-24} \mathrm{~g} / \mathrm{cm}^{3}}\right)\left(\frac{5 K}{T_{n}}\right)^{2} \times\left(\frac{\nu}{1 \mathrm{GHz}}\right)^{2}\left(\frac{Q_{W}}{Q_{A}}\right)
\end{aligned}
$$

with $T_{n}$ the total noise, $T_{n}=T_{\text {phys }}+T_{\text {elec }}$ (i.e the linear sum of cavity blackbody plus electronic noise temperatures) of the microwave detector

\subsection{First-Generation Experiments}

First-generation microwave cavity experiments of small volume were carried out at Brookhaven National Laboratory (BNL) and at the University of Flori- 
da (UF) They demonstrated the feasibility of the technique over a significant range of frequencies, but fell short in power sensitivity by $100-1000$ to detect halo dark matter axions of plausible model couplings

\subsection{The Rochester-Fermilab-Brookhaven (RBF) Experiment}

The experiment carried out at BNL was carried out by a collaboration of the University of Rochester, Fermi National Accelerator Laboratory and Brookhaven National Laboratory.[6] A superconducting solenoid magnet provided a peak central field of $85 \mathrm{~T}$ within the useful volume of roughly $15 \mathrm{~cm}$ diameter and $40 \mathrm{~cm}$ length. The cavity was constructed of high purity oxygenfree copper, for which the unloaded quality factor in the $2-3 \mathrm{GHz}$ range was $Q_{W} \sim 18 \times 10^{5}$ at physical temperature $4.2 \mathrm{~K}$, the operating temperature of the experiment. The cavity resonant frequency was tuned by means of a sapphire rod inserted longitudinally along the central axis of the cavity A schematic of the RBF experiment is shown in Figure 5.

The cavity field was sampled by a small coupling loop, isolated by a circulator, and fed to a cryogenic preamplifier Various amplifiers were used with $T_{\text {elec }}=$ $8-15 \mathrm{~K}$, the in situ noise calibration being performed by a noise source applied to a circulator at the amplifier input The amplified signal was mixed down in a double heterodyne receiver and fed to a 64 channel filter-detector system configured as a spectrum analyzer. Two sets of filters were used, one with $200 \mathrm{~Hz}$ bandwidth, the other with $400 \mathrm{~Hz}$ bandwidth. The 64 channel filter output was passed through a low-pass filter and the voltage digitized and recorded every $100 \mathrm{~ms}$ (the characteristic time of the low-pass filter) The 6 sets of cavity diameter and rod diameter combinations covered the frequency range $109-3.93 \mathrm{GHz}(4.5-16.3 \mu \mathrm{eV})$ A noteworthy detail among several innovations in this experiment was the use of a higher-TM mode to extend the frequency range of the search, albeit at the cost of a greatly reduced cavity form-factor $C$, and thus sensitivity.

The criterion for a candidate axion signal was a peak in the cavity output by a determined number of standard deviation above the local mean power Each frequency range was swept twice and a candidate peak was required to appear in both sweeps Ultimately no peaks survived re-examination. Limits (95\% c l) on $g_{A \gamma \gamma}{ }^{2}\left[\mathrm{GeV}^{-2}\right]$ were established of $57 \times 10^{-28}$ at the low mass end $(45 \mu \mathrm{eV})$ to $18 \times 10^{-25}$ on the high mass end $(16.3 \mu \mathrm{eV}$ ) (All reported microwave cavity results and projected future search regions are displayed together in Figure 6) 


\section{The University of Florida (UF) Experiment}

The other first-generation microwave cavity experiment was carried out at the University of Florida [7] The superconducting solenoid magnet $(171 \mathrm{~cm}$ diameter, $40 \mathrm{~cm}$ length) produced a peak central field of $8.6 \mathrm{~T}$ The cavities, made of high purity oxygen-free copper, had an unloaded quality factor at $15 \mathrm{GHz}$ of $Q_{W} \sim 16 \times 10^{5}$, measured at $42 \mathrm{~K}$ physical temperature The operating temperature of the experiment was normally $2.2 \mathrm{~K}$. Coarse tuning was done by a large dielectric rod moved transversely in the cavity, and fine tuning by insertion of a small tuning rod inserted through a hole in the top plate of the cavity.

Power from the $\mathrm{TM}_{010}$ mode of the cavity was extracted by a small loop, coupling to $H_{\phi}$. The cavity and the cryogenic preamplifier were isolated by a circulator The noise temperature of the HEMT (High Electron Mobility Transistor) amplifiers varied between 3-7 K, depending on frequency. A double heterodyne receiver mixed the amplified signal down to the $0-30 \mathrm{kHz}$ range, where a PC-based ADC/FFT calculated the power spectrum. The sampling rate of $70 \mathrm{kHz}$ was slightly greater than the Nyquist rate. Two cavities of different diameters, with both metal and dielectric tuning rods allowed the frequency range $132-1.83 \mathrm{GHz}$ to be covered completely

Unlike the RBF experiment, the UF experiment was step-tuned rather than sweep-tuned. In each 90 -second run, $10^{5}$ subspectra were averaged to generate a 32-bin power spectrum of $1 \mathrm{kHz}$ resolution. Any $2 \sigma$ peak above the local mean power was rescanned; this was repeated up to four times and persistent candidates were flagged for further investigation later: none survived Limits (975\% c.l.) on $g_{a \gamma \gamma}{ }^{2}\left[\mathrm{GeV}^{-2}\right]$ were established of $33 \times 10^{-28}$ at the low mass end $(5.46-5.95 \mu \mathrm{eV})$ to $12 \times 10^{-27}$ on the high mass end $(7.46-7.60 \mu \mathrm{eV})$. Noteworthy in the UF effort was extensive electromagnetic modeling of the microwave cavities and exploration of concepts for much higher frequency resonators [49] In particular, power-combining of two independent cavities was demonstrated at $300 \mathrm{~K}$, as a first step towards multiplexing many small cavities of high frequency while fully utilizing a large magnetic volume.[49]

\section{Second-Generation Experiments}

In the past five years there have been two new experiments, with the aim of finally achieving the requisite sensitivity to find or exclude halo axions with KSVZ or DFSZ couplings. The first of them represents a significant scale-up of previous microwave cavity experiments, and utilizes conventional HEMT microwave amplifiers. The second is a small-volume experiment, but utilizes a novel Rydberg-atom single-quantum detector instead of a conventional RF 
receiver to sample the cavity energy

\section{The US Large-Scale Experiment}

A large-scale microwave cavity experiment (dubbed the "US Large-Scale Axion Search") has been designed, constructed and operated by a collaboration of Lawrence Livermore National Laboratory (LLNL), the Massachusetts Institute of Technology (MIT), the University of Florida, Lawrence Berkeley National Laboratory (LBNL), Fermi National Accelerators Laboratory (FNAL), the University of Chicago, and the Institute for Nuclear Research/Moscow The experiment is sited at LLNL Drawing from the experience of the RBF and UF experiments, the key goals of this effort are (i) to achieve a power sensitivity required to see KSVZ axions constituting our halo, and (ii) to search a decade of mass range $13 \mu \mathrm{eV}<m_{A}<13 \mu \mathrm{eV}$ The increase in sensitivity largely accrues by an increase in magnetic volume $\left(B_{0}{ }^{2} V \sim 11 \mathrm{~T}^{2} \mathrm{~m}^{3}\right.$, compared to 036 and 045 for RBF, UF respectively), as well as considerable progress in HEMT amplifier development The increase in mass range will result from the implementation of multiple cavity arrays

\section{Experimental Description}

Figure 7 shows a schematic of the experiment The solenoid magnet $(60 \mathrm{~cm}$ diameter $\times 100 \mathrm{~cm}$ long) has an $8 \mathrm{~T}$ maximum field on-axis, but is normally run at $76 \mathrm{~T}$ The cavity $(50 \mathrm{~cm}$ diameter $\times 100 \mathrm{~cm}$ long) is made of - stainless steel, plated with high purity copper and annealed It is tuned by two independently-controlled tuning rods (copper or low-loss alumina) which may be moved radially between the wall and the center of the cavity, covering a range between $300-900 \mathrm{MHz}$ in $\sim 2 \mathrm{kHz}$ steps. Typically the loaded cavity quality factor $Q_{L} \sim 70,000$. A Joule-Thompson valve cools the cavity and amplifier to $\sim 13 \mathrm{~K}$. Normally the cavity is operated at a near-vacuum $(\sim 1$ Torr He) to avoid frequency drifts usually associated with backpressure fluctuations on bath helium

Power is extracted through an adjustable antenna inserted in the top plate of the cavity (coupling to $E_{z}$ ), a second weakly-coupled port allows the insertion of diagnostic signals Another test input is provided by the third port of a directional coupler between the cavity and the cryogenic preamplifier The signal from the electric field probe is amplified by two cryogenic HEMT amplifiers in series built by the National Radio Astronomy Observatory The combined power gain of the two cryogenic amplifiers is $\sim 34 \mathrm{~dB}$ Over the last two years the noise temperature of successive versions of these amplifiers improved from slightly over $4 \mathrm{~K}$ to below $2 \mathrm{~K}$ The axion receiver (Figure 8 ) first mixes the amplified signal down to an intermediate frequency (IF) of $107 \mathrm{MHz}$ After passing through a $35 \mathrm{kHz}$ wide IF filter, a second stage mixes the signal 
down to audio frequency (AF) of bandwidth $50 \mathrm{kHz}$ centered at $35 \mathrm{kHz}$ At this point the signal bifurcates into two data streams In the first, the AF signal is sent to a commercial fast Fourier transform instrument which computes a power spectrum of 400 channels each of width $125 \mathrm{~Hz}$. More precisely, this power spectrum results from an equal-weight average of 10,000 subspectra, each representing $(125 \mathrm{~Hz})^{-1}=8 \mathrm{msec}$ of integration time. This is the 'medium-resolution' data $\Delta E / E \sim \mathcal{O}\left(10^{-7}\right)$, for which the virialized component would appear as a peak of approximately 6 channels width. The second stream results in a power spectrum calculated by an on-board FFT from a single 50-second long integration within the 80-second run at each frequency. This yields the 'high resolution' data $0.02 \mathrm{~Hz}, \Delta E / E \sim \mathcal{O}\left(10^{-11}\right)$ which is used to look for narrow peaks resulting from recent infall axions which have not yet thermalized, as discussed above

\section{Data Collection and Analysis}

As mentioned previously, in this experiment the cavity frequency was shifted by $\sim 2 \mathrm{kHz}$ after each 80 second run, or approximately $1 / 15$ of the cavity bandwidth. The entire frequency range was swept in this manner at least three times to achieve the required sensitivity, as well as reduce systematic errors. Thus each frequency was included in $\sim 50$ subspectra, which were then combined with appropriate weighting into a continuous power spectrum over the broad frequency interval with $125 \mathrm{~Hz}$ bin width Small holes in the frequency coverage due to avoided crossings between the $\mathrm{TM}_{010}$ mode and intruder $\mathrm{TE}$ or TEM modes were later filled in by flooding the cavity with superfluid helium ( $n=1.055)$ which shifted the mode-crossing away from the frequency region of interest

For the medium-resolution data, candidates are identified by excess power in one bin $(125 \mathrm{~Hz})$, and six bins $(750 \mathrm{~Hz})$ For these candidate bins, a second independent data set is acquired at the same signal-to-noise ratio. This new data is combined with the old and any frequencies that show persistent power excess are logged for further examination. A third set of independent data is acquired, combined with the previous two data sets, and the number of candidates now is tractable for each to be examined individually. The few very strong external radio sources mimicking persistent candidates are easily identified and rejected Should any persistent candidates remain even after further running, the ultimate test would be to examine whether the signal varies as $B^{2}$, and specifically whether it vanishes when the field is turned off Monte Carlo simulation techniques are used to determine the sensitivity and confidence level of the six-bin search path

The high resolution data were analyzed similarly, the data are binned on-line at resolutions of $0.02,016$, and $128 \mathrm{~Hz}$, and candidates defined as those peaks exceeding $15 \sigma, 8 \sigma$, and $5 \sigma$ respectively 
A critical check for the experiment is shown in Figure 9, which is a histogram of the dispersion of the combined single bin data, with the power excess plotted in units of the $\mathrm{r}$ m.s deviation about the mean power The smooth curve is the distribution based on Gaussian thermal noise treated as input to the analysis chain That this experiment was in fact sensitive to KSVZ axions is shown in Figure 10 which shows the expected signal $\left(\sim 3 \times 10^{-23} \mathrm{~W}\right)$ relative to the noise background with one- and six-channel binning.

\section{Results}

This experiment has recently reported results[8] over the frequency range 701$800 \mathrm{MHz}$, corresponding to a range of axion masses of $290-331 \mu \mathrm{eV}$ There were no 6-bin candidates surviving all scans. Furthermore, from the high resolution search, upper limits on axion power were established at approximately $33 \times 10^{-23} \mathrm{~W}(002 \mathrm{~Hz}), 50 \times 10^{-23} \mathrm{~W}(016 \mathrm{~Hz})$, and $88 \times 10^{-23} \mathrm{~W}(128 \mathrm{~Hz})$. This analysis also searched for coincidences between the high- and mediumresolution data, again with null results.

Figure 6 shows the axion-photon couplings excluded by the US large-scale axion search and earlier RBF and UF experiments (Note that as $g_{A \gamma \gamma} \propto$ $m_{A}$ the presentation $\left[g_{A \gamma \gamma} / m_{A}\right]^{2}$ has the effect of flattening the graph.) For all results, the results are normalized to $90 \% \mathrm{cl}$., and the best maximumlikelihood estimate of the halo CDM density $\rho_{C D M}=75 \times 10^{-25} \mathrm{~g} / \mathrm{cm}^{3}$ is used [50]

At the time of writing this effort has extended the exclusion range to $275 \mu \mathrm{eV}<$ $m_{A}<335 \mu \mathrm{eV}$ at approximately the same sensitivity level, and continues to run Of equal significance was that the sensitivity of the microwave cavity experiment was finally brought into the region of interest, $i e$, where the axion could plausibly be discovered.

\section{Future Prospects-DC SQUID Amplifiers}

There is a high premium on noise temperature in the Sikivie microwave cavity experiment For a fixed signal-to-noise and search rate, the axion-photon coupling that can be reached $g_{A \gamma \gamma}^{2} \propto T$; alternatively for a fixed axion model, the frequency search rate $d \nu / d t \propto T^{-2}$

Two areas of active development towards lower noise temperature for the axion experiment have been DC SQUID amplifiers and Rydberg atom singlequantum detectors The first behave as classical coherent amplifiers, and are therefore ultimately subject to the quantum limit in noise temperature, $T_{\text {lim }}=$ $h \nu / k_{b}, \sim 50 \mathrm{mK}$ at $1 \mathrm{GHz}$. The second are "RF phototubes," and being phaseless single-quantum detectors evade the quantum limit. DC SQUIDs are the technology choice for upgrade of the US large-scale experiment, and will be briefly described now Rydberg atom single-quantum detection is the technol- 
ogy of the Kyoto microwave cavity axion search and will be discussed in the next section.

A DC SQUID (shown schematically in Figure 11) is a loop of superconductor broken in two places by Josephson junctions. Bias current is applied across the loop and the output voltage across the junctions is a sinusoidal function of the magnetic flux threading the loop.[51] As an amplifier, the input signal is converted to magnetic flux by an input coil and the varying flux modulates the output voltage. Conventionally the construction of DC SQUID amplifiers has been a square pancake-coil primary lying on top of the superconducting singleturn secondary (the "washer"), about a millimeter on a side. Until recently, such devices have been used for low noise amplification only up to frequencies of $250 \mathrm{MHz}$ or so Above that frequency, the gain dramatically rolled off due to the parasitic capacitance between the input coil and SQUID substrate

A recent breakthrough in coupling the input signal to the SQUID has dramatically increased the useful frequency of these devices.[52] In this scheme, the ground return connection of the input coil has been broken, resulting in a stripline resonator with $\sim 7 \Omega$ stripline impedance and $Q \sim 7$ for $50 \Omega$ impedance to the SQUID The resonance is at the detuned frequency where the parasitic capacitance is canceled by the resonator reactance Figure 12 shows the device gain at several different input tunes, and Figure 13 shows the SQUID noise temperature at 42 and $18 \mathrm{~K}$ bath temperature

\subsection{The University of Kyoto Experiment}

Another second-generation axion search is under development at the University of Kyoto This effort seeks to exploit the extremely low-noise photon counting capability of Rydberg atoms in a Sikivie-type microwave cavity experiment. The initial goal is to sweep out a $10 \%$ mass window around $24 \mu \mathrm{eV}$

\section{Rydberg-Atom Single Quantum Detection}

Rydberg atoms are atoms (usually alkali metals) where one electron is promoted to a principal quantum number $n>>1$, near the ionization limit [53] The valence electron of such highly excited atoms is hydrogen-like These Rydberg atom states for $n \sim \mathcal{O}(100)$ are promising as microwave-photon detectors for three reasons: (i) The energy difference between adjacent levels $\left(E_{n+1}-E_{n}\right)$, which scales as $n^{-3}$, is in the microwave region. For $n \approx 100$ the energy difference is $\approx 7 \mathrm{GHz}$ (ii) The electric dipole transition rate between adjacent levels, which scales as $n^{2}$, is large, implying a high efficiency for absorbing a microwave photon For $n \approx 100$ the transition rate is $3 \times 10^{4} / \mathrm{s}$ (iii) The lifetime of excited states, which scales as $n^{3}$, is suitably long For $n \approx 100$ the lifetime is $\approx 1 \mathrm{~ms}$ 
The use of Rydberg atoms for single-quantum counting is an idea going back more than two decades,[54] and suggests the following implementation for the Sikivie axion experiment The "front-end" of the experiment is the same, $i e$ there is a tunable cavity for axion-photon conversion Photons from this cavity are coupled out to a field-free antechamber, also tuned to the same frequency An atomic beam is prepared to the desired Rydberg level by optical pumping, which then traverses the antechamber Here, however, the spacing of the transition levels must be adjusted to match the frequency of the cavity, this may be accomplished through the Stark effect, by applying a small DC voltage in a parallel-plate configuration. It is here that the Rydberg atoms absorb the microwave photons.

Upon exiting the chamber, the atomic beam is now a mixture of two states, with most of the atoms in the prepared state, and very few atoms promoted to the higher Rydberg level Detection of only those atoms which have absorbed the microwave photon of the correct frequency is accomplished by selective field ionization After exiting the chamber, the beam passes through a uniform electric field, perpendicular to its velocity, with a prescribed electric field The combined Coulomb plus linear potential has a saddle point, or potential barrier, whose height is set precisely (through the applied electric field) such that electrons promoted to the upper level are unbound, $i$ e. simply fall out of the atom, whereas those in the initial state remain bound. Just as in a photomultiplier tube, the free electron is then accelerated and detected. In practice, one may discriminate between energy levels differing by only a few 10 's of $\mathrm{MHz}$

There are several challenges in implementation At the most fundamental level, the quantum mechanical interaction of the Rydberg states with the cavity ("Cavity QED") is non-trivial Additionally, there are coherent interactions of Rydberg atoms in the cavity At the more practical level, the "turn-on" of fields seen by the atoms must be adiabatic so as not to project the many Rydberg states onto several Stark states. Similarly, the Rydberg states are only weakly bound and slight perturbations may induce non-selective ionization Lastly, the energy (frequency) resolution with which an axion signal may be resolved is limited by the transit time of the atoms through the cavity, and would seem to be marginal for the virialized component Sensitivity to finestructure does not seem possible

\section{Experimental Description}

An experiment utilizing Rydberg atom single-quantum detection in Kyoto is well-along in commissioning ('CARRACK', for Cosmic Axion Research with Rydberg Atoms in a Resonant Cavity in Kyoto) [9] A sketch of the apparatus is shown in Figure 14 The microwave resonator is a single copper cavity (45 cm radius, $725 \mathrm{~cm}$ long) which fits inside a superconducting solenoid 
(15 cm diameter, $50 \mathrm{~cm}$ long, $7 \mathrm{~T}$ peak field) Power from the conversion cavity is coupled to a niobium superconducting cavity just above it, where the magnetic field is canceled by a bucking coil The frequency of both cavities are made to track by means of $6 \mathrm{~mm}$ sapphire rods inserted axially into them The cavities are cooled to $<15 \mathrm{mK}$ by means of a dilution refrigerator.

A beam of rubidium atoms is accelerated, neutralized and directed vertically through the detection cavity Just before entering the detection cavity, the atoms are excited to a Rydberg state with principal quantum number near 160 , by triple optical excitation with three colinear diode laser beams. In the detection cavity, the Rydberg atoms are then Stark-tuned so an E1 $n p \rightarrow(n+2) s$ transition is matched to the cavity frequency After exiting, the Rydberg atoms are selectively ionized by an electric field (around $0.5 \mathrm{~V} / \mathrm{cm}$ ) and the liberated electron is detected and amplified by an electron multiplier (a "Channeltron").

\section{Present Status}

Studies have been performed to confirm that the experiment is sensitive to single blackbody photons in the $<15 \mathrm{mK}$ range. These include verifying the temperature dependence, and the number and velocity of the Rydberg atoms [55] Several percent of mass range around $24 \mathrm{GHz}(\sim 10 \mu \mathrm{eV})$ has been swept out, but candidate peaks have not been eliminated yet, nor have potential systematic backgrounds been rejected

\section{Other Methods for $m_{A}<\mathcal{O}(1 \mathrm{eV})$}

In this section, we review experiments which set limits on the axion-photon coupling, but do not depend on the axion being our halo dark matter. Searches for photon regeneration and vacuum birefringence are purely laboratory experiments and require no astrophysical or cosmological assumptions The search for solar axions does depend on axion production in the Sun's core, but the calculated flux is robust and does not depend on subtle details of the solar model Axion-photon mixing is common to the last three, for which we will give a brief introduction A telescope search for $A \rightarrow \gamma \gamma$ from the halos of galactic clusters has set interesting limits in the $3-8 \mathrm{eV}$ range. Finally, as a light pseudoscalar would imply a long-range force, we review the experiments setting limits on axion-like particles by searches for new macroscopic forces at the $\mathcal{O}(01-10) \mathrm{cm}$ scale 


\section{Axion-Photon Mixing}

The axion, having a two-photon coupling, can mix with photons in an external electromagnetic field via a Primakoff interaction. More specifically, the electromagnetic field can be represented as a sea of virtual photons, and the interaction between a real photon and a virtual photon can produce a real axion (Figure 15a). Likewise, a virtual axion may be created, contributing to a non-trivial index of refraction, $|n-1|>1$ (Figure 15b) Higher-order QED itself already provides for such an index (Figure 15c), and as will be seen both axions and the four-photon diagram make the vacuum birefringent $\left(n_{\perp} \neq n_{\|}\right)$ The presence of a background gas or plasma also enter into the axion-photon mixing, as it modifies the dispersion relationship for the real photon. All of these effects have been the basis for experiments searching for the axion or higher-order QED.

Axion-photon mixing in full generality (and photon mixing with other lowmass particles, e.g. the graviton) has been carried out by Raffelt and Stodolsky [56] This formalism is most useful for problems where the axion is relativistic, such that the axion and photon are nearly degenerate. For a photon in a field-free vacuum, $k=\omega$. The momentum difference between an axion and a photon of the same energy is $\Delta_{A}=k_{A}-\omega=-m_{A}{ }^{2} / 2 \omega$. If the external electromagnetic field is a transverse magnetic field $\mathrm{B}$, then only photons polarized with $\mathbf{E} \| \mathbf{B}$ can couple to axions, as the effective Lagrangian is given by $\mathcal{L}=g_{A \gamma \gamma} \phi_{A} \mathbf{E} \cdot \mathbf{B}$. As mentioned above, the vacuum itself is birefringent in a magnetic field, with $\Delta_{\perp}=(4 / 2) \omega \xi \sin ^{2} \Theta$ and $\Delta_{\|}=(7 / 2) \omega \xi \sin ^{2} \Theta$ Here $\xi=(\alpha / 45 \pi)\left(B / B_{\text {crit }}\right)^{2}$, where the critical field strength is $B_{\text {crit }}=m_{e}^{2} / e \sim 4.41 \times 10^{13} \mathrm{G}$, and $\Theta$ is the angle between the photon momentum and the direction of the external field. Indices of refraction $k_{j}=n_{j} \omega$ are then given as $n_{j}=1+\Delta_{j} / \omega$ The linearized wave equation is then

$$
\left[\omega+\left(\begin{array}{ccc}
\Delta_{\perp} & 0 & 0 \\
0 & \Delta_{\|} & \Delta_{g} \\
0 & \Delta_{g} & \Delta_{A}
\end{array}\right)-i \frac{\partial}{\partial z}\right]\left(\begin{array}{c}
A_{\perp} \\
A_{\|} \\
A
\end{array}\right)=0
$$

where $A_{\perp}, A_{\|}$are the amplitudes of the two photon polarization states, and $A$ that of the axion. The off-diagonal component is $\Delta_{g}=(g B / 2) \sin \Theta$ and represents the axion-photon mixing For simplicity, $g=g_{A \gamma \gamma}$ hereafter

It should be noted that in a vacuum, axions and photons cannot be made degenerate as $\Delta_{A}<0$ and $\Delta_{t, \|}>0$. As will be seen in the search for solar axions however, in the $\mathrm{x}$-ray regime one may give the photon an effective mass in a medium, thus allowing the axion and photon to be made degenerate. 
The combination of modern laser technology with very long high-field dipole magnets developed for high energy physics colliders made it possible to think about purely laboratory searches for the invisible axion Two such proposals were published in the mid-1980's and were carried through to experiments

\subsubsection{Photon Regeneration ("Shining Light through Walls")}

Working to second order and diagonalizing the lower part of Eqn. 9 above, the mixing strength is characterized by (1/2) $\tan 2 \theta=\Delta_{g} /\left(\Delta_{\|}-\Delta_{A}\right)$. The weakmixing case corresponds to $(1 / 2) \tan 2 \theta \sim \theta$, i e $\theta<<1$, which will always pertain for these experiments (N.B: Neither $\Theta$ nor $\theta$ are to be confused with the QCD $\Theta$ parameter ) Consider now a photon which enters a region of uniform transverse magnetic field, and whose polarization is aligned with the field direction. The transition probability that the photon is in the axion state at any point $z$ along the magnet is given by

$$
\Pi(\gamma \rightarrow A)=4 \theta^{2} \sin ^{2}\left(\Delta_{\mathrm{osc}} z / 2\right)
$$

As expected, the momentum mismatch between the photon and axion, $\Delta_{\mathrm{OSC}}=$ $\Delta_{\|}-\Delta_{A}$ defines an oscillation length, $l_{\text {osc }}=2 \pi \Delta_{\text {OSc }^{-1}}$ such that at multiples of that distance the mixed state is a photon again with unit probability The transition probability can be put in a more explicit form

$$
\Pi(\gamma \rightarrow A)=(1 / 4)(g B z)^{2} F^{2}\left(\Delta_{\mathrm{osc}} z\right)
$$

where $F\left(\Delta_{\mathrm{osc}} z\right)=\left[\sin \left(\Delta_{\mathrm{osc}} z / 2\right) /\left(\Delta_{\mathrm{osc}} z / 2\right)\right]$. For all situations of practical concern, $\Delta_{\text {osc }} \sim-\Delta_{A}=m_{A}{ }^{2} / 2 \omega$. The function $F^{2}$ is thus unity for all axion masses such that the length of the magnetic field region $\Delta_{\mathrm{osc}} z<<2 \pi$, but oscillates and falls off rapidly for larger masses.

That a beam of photons can convert into a beam of axions suggests a Hertzian experiment with a symmetrical arrangement of two colinear dipole magnets with an interposing barrier, as shown in Figure 16a.[57] A laser beam is directed down the bore of the first, and a low-noise detector for single photons is put at the end of the second. Axions created in the first magnet traverse the wall, and can convert back with the same probability, i.e., $p(\gamma \rightarrow A, A \rightarrow$ $\gamma)=\Pi^{2} \propto g^{4}$ To build up the effective laser power in the first dipole, one would make an optical cavity or ring laser. The low-noise detector of choice for a laser in the optical would be a cooled spot-cathode photomultiplier tube Describing the magnets by a length $l$ and a strength $\mathrm{B}$, the laser by a power $\mathrm{P}$, frequency $\omega$, and duty-factor $\delta$, and the photon detector by its efficiency $\epsilon$ and noise rate $\nu$, then the sensitivity in $g$ achievable in an integration time $t$ 
is given by Eqn 12 .

$$
\begin{aligned}
g<\left(47 \times 10^{-9} \mathrm{GeV}^{-1}\right) & {\left[\frac{10 \mathrm{~T}}{B_{0}} \frac{10 \mathrm{~m}}{l}\right]\left[\frac{\omega}{25 \mathrm{eV}} \frac{1 \mathrm{~kW}}{P} \frac{01}{\epsilon}\right]^{1 / 4} } \\
\times & {\left[\frac{100 \mathrm{~d}}{t} \frac{\nu}{001 \mathrm{~Hz}} \frac{\delta}{10 \times 10^{-4}}\right]^{1 / 8} }
\end{aligned}
$$

The value of $g$ attainable in a simple photon regeneration experiment is shown as the dashed line in Figure 17, when the benchmark values for the experimental parameters in Eqn. 12 are all met While the sensitivity compares favorably with the solar evolution limit, it should be clear that the experiment is not readily improved, as $g$ only decreases as $P^{-1 / 4}$, and $t^{-1 / 8}$ The limitations due to external sources of noise may be eliminated by homodyning, by which part of the incident light is sent around the barrier, and recombined with the optical path corresponding to the amplitude for $(\gamma \rightarrow A, A \rightarrow \gamma)$ (Figure 16b) The knee in the curve of sensitivity vs. mass may be also extended upwards somewhat by making a 'wiggler' of dipole magnets of alternating polarity. Nevertheless, we are not able to see how a photon regeneration experiment could be augmented or scaled to reach the usual axion models.

The basic photon regeneration experiment has been carried out by Ruoso et al [58], using two Brookhaven CBA dipoles (4.4 m each, $37 \mathrm{~T}$ ), an argon-ion laser ( $1.5 \mathrm{~W}, 200$ traversals in an optical cavity), and a special low dark current phototube. A limit of $g<77 \times 10^{-7} \mathrm{GeV}$ was established, for $m_{A}<10^{-3} \mathrm{eV}$

\subsection{Polarization Effects}

The existence of pseudoscalars may also be demonstrated by two effects on the polarization state of light traveling through a transverse magnetic field [59] Consider a beam of plane-polarized light incident on a dipole magnet, such that its polarization vector is at an angle $\varphi$ with respect to the magnetic field The first effect is due to the attenuation of photons polarized parallel to the magnetic field, due to the production of axions (Figure 15a) This results in the rotation of the plane of polarization (Figure 18a), since the $E_{\|}$component can produce axions, $E_{\| l}$ would be foreshortened by a tiny amount relative to the $E_{\perp}$, which cannot produce axions. It is easily seen that the angle of rotation is given by

$$
\epsilon=(1 / 4) \sin 2 \varphi \quad \Pi(a \rightarrow \gamma) \sim(1 / 16) \quad \sin 2 \varphi \cdot(g B l)^{2}
$$

where the latter approximation obtains for $m_{A}$ sufficiently light that $\Delta_{\text {osc }} l<<$ $2 \pi$. For the special case of the incident light being polarized at $45^{\circ}$, the angle of rotation maximizes at $\Pi / 4$. The rotation angle builds up linearly with the 
number of bounces $N$ if an optical cavity inside the magnet is used. The second effect is vacuum birefringence, $i e$. the conversion of linearly polarized light into elliptically polarized light, due to the phase difference between the parallel and perpendicular components of the light in a vacuum (Figure 18b) Here however, there are three distinct contributions. One contribution to the phase difference is due to the production of virtual axions (Figure 15b), the phase difference results as axions are massive and the mixing occurs only within the parallel polarization state The axionic contribution to the phase difference is given by

$$
\begin{array}{r}
\phi_{A}=\theta^{2} \cdot\left[\left(\Delta_{\mathrm{osc}} l / 2\right)-\sin \left(\Delta_{\mathrm{osc}} l / 2\right)\right] \\
\phi_{A} \sim(1 / 48)\left(g B m_{A}\right)^{2} l^{3} / \omega
\end{array}
$$

the approximation Eqn. 15 holding with the same proviso on the axion mass Second, QED by itself predicts vacuum birefringence (Figure 15c); from the parallel and perpendicular indices of refraction at the outset of this section, the phase difference is

$$
\phi_{\mathrm{QED}}=(-2 / 15) \quad\left(\alpha B / m_{e}^{2}\right)^{2} \omega l
$$

Finally, there is the birefringence associated with residual gas, the CottonMouton effect

$$
\phi_{\mathrm{CM}}=2 \pi C B^{2} l
$$

where $C$ is a constant depending on the species of gas Within an actual experiment, the ellipticity depends on the total phase difference, which is the sum of all three contributions, $\phi=\phi_{A}+\phi_{\mathrm{QED}}+\phi_{\mathrm{CM}}$. Furthermore, the total phase difference accumulated may be enhanced by means of an optical cavity within the magnet, which makes $\phi_{\mathrm{Tot}}=N \phi$. Note that for the axionic contribution, this is not equivalent to a magnet of length $L=N l$, since the axion-photon coherence is lost at each mirror reflection What is actually measured is the ellipticity, the ratio of the minor to the major axis of the elliptically polarized light, which in the limit of small $\phi$, is given by $\Psi=|\phi| \sin 2 \varphi / 2$. (In actual practice, ellipticity is converted into polarization rotation by inserting a $\lambda / 2$ plate before the final polarizer) Once again, the observable is maximum at an incident polarization angle of $\varphi=45^{\circ}$, where $\Psi=|\phi| / 2$ Such an experiment has been carried out by a Rochester-BNL-FNAL collaboration at Brookhaven, $[60]$ as shown schematically in Figure 19 Light from argon-ion laser ( $2 \mathrm{~W}$ in one line at $5145 \mathrm{~nm}$ ) is polarized at a $45^{\circ}$ angle to the magnetic field direction, and injected into an optical cavity within two superconducting dipole magnets placed end-to-end. These magnets were $44 \mathrm{~m}$ long, with a maximum magnetic field of $5 \mathrm{~T}$. After the beam exits the optical cavity having made several hundred traversals, it enters the ellipsometer where the light is analyzed by a polarizer orthogonal to the incident light, and the transmitted 
intensity recorded by a photodiode The usual strategies are employed to enhance the signal-to-noise ratio, $i$ e the polarization before the final analyzer is modulated at a frequency $\omega_{f} / 2 \pi=312.5 \mathrm{~Hz}$ so the signal appears as a linear cross-term rather than quadratically, furthermore, the magnetic field was modulated at a frequency $\omega_{m} / 2 \pi=0.032 \mathrm{~Hz}$ to move the signal out to sidebands at $\omega_{f} \pm \omega_{m}$

The expected signal level under any scenario is exceedingly small For typical values in this experiment, $\left\langle B_{\text {ext }}\right\rangle^{2}=45 \mathrm{~T}^{2}, N=500$, the QED-induced ellipticity $\Psi^{\mathrm{QED}}=47 \times 10^{-13}$ The axion-induced signals for a standard axion-photon coupling are much smaller For example, for $m_{A} \sim 10^{-3} \mathrm{eV}$, where $g \sim 10^{-13} \mathrm{GeV}^{-1}, \Psi^{A}=3 \times 10^{-22}$ and $\epsilon=2 \times 10^{-25}$ The identification and mitigation of the noise sources in this experiment is too large a topic for this review, suffice to say that subtle instrumental effects dominated over the limits expected from shot noise. In the final analysis, the noise floor was further reduced by vector-averaging the data from several runs, and utilizing the fact that the phase of the magnetic field modulation is known. The best sensitivities for the optical rotation and ellipticity were $\epsilon=0.38 \times 10^{-9}$ and $\Psi=20 \times 10^{-9}$ respectively, at the $95 \% \mathrm{cl}$. from which were derived the exclusion regions of Figure $20\left(M=1 / g_{A \gamma \gamma}\right)$ Note that the optical rotation data produces the better limit at lower masses $\left(m_{A}<8 \times 10^{-4} \mathrm{eV}\right)$, and the ellipticity data at the higher masses While the sensitivity of this experiment is hopelessly far away from that required to see standard axions, it came within 4 orders of magnitude of the QED contribution

- There is a new experiment in construction at Fermilab, by a Colorado StateNIST-FNAL collaboration.[61] Many improvements are being incorporated, most importantly the use of two SSC dipoles $(66 \mathrm{~T}$ peak field, $17 \mathrm{~m}$ long each), a Fabry-Perot cavity of high finesse $\left(\mathcal{O}\left(10^{5}\right)\right)$ rather than an optical delay line, and LIGO-technology active stabilization. It is claimed that this experiment should be able to set limits on the axion-photon coupling $g_{A \gamma \gamma}<$ $1.5 \times 10^{-10} \mathrm{GeV}^{-1}$, comparable to the red giant limit, and measure for the first time the QED vacuum polarization at the $05 \%$ level.

\section{Cosmological/Astrophysical axions}

While it now seems sure that the limits on axions from globular cluster stars and SN1987a overlap completely, historically there had been a concern over the possibility of a ' $1 \mathrm{eV}$ window' where axions may not have been excluded This motivated two lines of work in this mass region, one a telescope search for a quasi-monochromatic line from the spontaneous decay of thermally-produced axions in clusters of galaxies, and the other a search for solar axions by their conversion to x-rays in a magnetic field. 


\section{Telescope Search for Decaying Relic Thermal Axions}

For axions of mass greater than about $10^{-1} \mathrm{eV}$, their cosmological abundance is no longer dominated by vacuum misalignment or string radiation mechanisms, but rather by thermal production Their contribution to the critical density is small, $\Omega_{A} \sim 001 m_{A}[\mathrm{eV}]$ However, the spontaneous decay lifetime of axions, $\tau(A \rightarrow 2 \gamma) \sim 10^{25} \sec \left(m_{A}[\mathrm{eV}]\right)^{-5}$ while irrelevant for $\mu \mathrm{eV}$ axions, is short enough to afford a powerful constraint on such thermally produced axions in the $\mathrm{eV}$-range, by looking for a quasi-monochromatic photon line from galactic clusters This line, corrected for Doppler shift, would be at half the axion mass and whose breadth would be consistent with the observed cluster internal virial motion, typically $\Delta \lambda / \lambda \sim 10^{-2}$ The conservative assumption is made that the relative density of thermal axions fallen into the cluster gravitational potential reflects their overall cosmological abundance A search for thermal axions in three rich Abell clusters was carried out at Kitt Peak National Laboratory [62] The search strategy was to record the spectrum while sighting at the center of a cluster, and then recording the spectrum several core radii off-center. By properly normalizing and subtracting, one may eliminate the atmospheric molecular emission lines which crowd the spectra. For the three clusters studied (all with $z=006-0.17, \sigma \sim 1300 \mathrm{~km} / \mathrm{sec}$, and core radius $a \sim$ $400 \mathrm{kpc}$ ), the expected line intensity for DFSZ axions would be on the order $I_{A} \sim 10^{-17}\left[m_{A} /(3 \mathrm{eV})\right]^{7} \mathrm{erg} \mathrm{cm}^{-2} \operatorname{arcsec}^{-2} \AA^{-1} \mathrm{~s}^{-1}$, comparable to the continuum night sky emission. (Five powers of the mass are due to the lifetime, one due to the cosmic abundance, and one due to the energy of the photon ) No such line was observed between $3100-8300 \AA\left(m_{A}=3-8 \mathrm{eV}\right)$ after "on-off field" subtraction (Figure 21).-The exclusion limit improves with mass, going from $g_{A \gamma \gamma}<75 \times 10^{-11} \mathrm{GeV}^{-1}$ at $m_{A}=3.5 \mathrm{eV}$, and $g_{A \gamma \gamma}<1.27 \times 10^{-12} \mathrm{GeV}^{-1}$ at $m_{A}=75 \mathrm{eV}$.

\section{Searches for Solar Axions}

Axions produced within our Sun's interior by a Primakoff process also present an ideal opportunity to carry out a search for $\mathcal{O}(\mathrm{eV})$ axions, by looking for their reconversion to $\mathrm{x}$-rays in a large magnetic field. [5,63] The calculated axion spectrum is shown in Figure 22, and the total flux at the Earth of $\sim 10^{12} \mathrm{~cm}^{-2} \mathrm{sec}^{-1} m_{A}[\mathrm{eV}]^{2}$ is not sensitive to details of the solar model Unfortunately, their average energy is $\sim 4 \mathrm{keV}$, implying an oscillation length in vacuum of $2 \pi\left(m_{A}^{2} / 2 \omega\right)^{-1} \sim \mathcal{O}(\mathrm{mm})$, seemingly precluding the mixing from achieving its theoretically maximum value in any practical magnet However, one can endow the photon with an effective mass in a gas, thus permitting the axion and photon dispersion relationships to be matched [63] Recalling that for x-rays, $n=1-m_{\gamma}^{2} / 2 \omega^{2}$, a gas pressure may be found such that $m_{\gamma}^{2}=\omega_{\mathrm{pl}}^{2}=4 \pi r_{0} \rho f_{1}$, where $r_{0}$ is the classical electron radius, $\rho$ the density, and $f_{1} \sim Z$ the atomic scattering factor. The mass range for the axion is swept 
out by varying the gas pressure The original concept was a stationary large volume solenoidal magnet filled with the dispersion-matching gas, and lined with a thin-window multiple-wire proportional chamber array for detection of the x-rays (Figure 23) However, the first implementation of this proposal was carried out using a simple dipole magnet ( $18 \mathrm{~m}$ long, $15 \mathrm{~cm}$ bore, $22 \mathrm{~T}$ ) with a conversion volume of variable pressure helium gas, and a xenon proportional chamber as the $\mathrm{x}$-ray detector.[64] The magnet was fixed in orientation to take data for $\sim 1000 \mathrm{sec} /$ day, as the Sun crossed the horizon at sunset Running with three different gas pressures, axions were excluded for $g_{A \gamma \gamma}<36 \times 10^{-9} \mathrm{GeV}^{-1}$ for $m_{A}<003 \mathrm{eV}$, and $g_{A \gamma \gamma}<77 \times 10^{-9} \mathrm{GeV}^{-1}$ for $003 \mathrm{eV}<m_{A}<011 \mathrm{eV}$ $(997 \% \mathrm{c} 1)$, see Figure 24 A more ambitious experiment, using a superconducting magnet on a telescope mount to track the Sun continuously has reported a preliminary exclusion limit of $g_{A \gamma \gamma}<6 \times 10^{-10} \mathrm{GeV}^{-1}(95 \% \mathrm{cl})$ for $m_{A}<003 \mathrm{eV}[65]$

Another search for solar axions is underway (SOLAX), using a single crystal germanium detector It exploits the coherent conversion of axions into photons when their angle of incidence satisfies a Bragg condition with a crystalline plane Analysis of $194 \mathrm{~kg} \cdot \mathrm{yr}$ of data from a $1 \mathrm{~kg}$ germanium detector yields a bound of $g_{A \gamma \gamma}<27 \times 10^{-9} \mathrm{GeV}^{-1}(95 \% \mathrm{cl}$.), independent of mass up to $m_{A} \sim 1 \mathrm{keV}[66]$

\section{New Macroscopic Forces}

Axions mediate a monopole-dipole interaction potential between spin and matter[67] given by

$$
V \propto g_{s} g_{p} \frac{\hat{\sigma} \cdot \hat{r}}{m_{p}}\left(\frac{1}{\lambda r}+\frac{1}{r^{2}}\right) e^{-r / \lambda}
$$

where $g_{s}$ and $g_{p}$ are coupling constants at the scalar $(s)$ and polarized $(p)$ vertices, $\vec{\sigma}$ and $\vec{r}$ the spin and separation vectors, $m_{p}$ the mass at the polarized vertex, and $\lambda$ the range of the force $\left(\propto 1 / m_{A}\right)$ For axions with mass within the window 1-1000 $\mu \mathrm{eV}$, the corresponding $\lambda$ is $02-200 \mathrm{~mm}$ The scalar coupling $g_{s}$ is proportional to the strong interaction CP violating parameter $\bar{\theta}$, and the product of couplings is

$$
g_{s} g_{p} \simeq \frac{\bar{\theta}}{\lambda[\mathrm{mm}]^{2}} 6 \times 10^{-27}
$$

Recall that null searches for a neutron edm conservatively imply $\vec{\theta}<10^{-9}$, and the axion resulted from a scheme to drive $\bar{\theta}$ to zero However, in the Standard Model plus axions, $\bar{\theta}$ does not identically vanish There remains a small contribution from known sources of weak interaction CP violation, at the 
level of perhaps $\vec{\theta} \simeq 10^{-14}[67]$ should weak interaction CP violation arise from the Kobayashi-Maskawa model Axions would be signaled by a weak Yukawatype gravitational force at short but macroscopic distances, with strength proportional to the CP violating parameter $\bar{\theta}$.

Two such recent null experiments placed upper limits on the product coupling $g_{s} g_{p}$ in a system of magnetized media and test masses. One experiment $[68]$ had peak sensitivity, relative to $\bar{\theta}$ fixed, near $10 \mathrm{~mm}$ (20 $\mu \mathrm{eV}$ axions), the other[69] had peak sensitivity near $100 \mathrm{~mm}(2 \mu \mathrm{eV}$ axions $)$ Both experiments lacked by 10 orders of magnitude or so the required sensitivity in $g_{s} g_{p}$ to detect axions at the $\bar{\theta}$ allowed by the neutron edm search experiments (see Figure 25) Two recent proposals aim to improve the overall sensitivity by several orders of magnitude[70] and improve the sensitivity at short distances, down to perhaps $1 \mathrm{~mm}(200 \mu \mathrm{eV}$ axions) However, these new experiments will likely lack by at least several orders of magnitude the sensitivity to $g_{s} g_{p}$ required by the small $\bar{\theta}$ implied by neutron edm limits

\section{Summary and Outlook}

While much has happened both in particle physics and cosmology over the past two decades, the motivation for the axion remains strong. It is still the most compelling mechanism to enforce Strong-CP conservation, and the sum total of all cosmological observables still point to a large CDM component in the Universe Serious efforts to discover the axion should therefore be a highpriority component of the program in particle astrophysics, and the state of technology is well-prepared for one or more major experiments

Figure 26 shows all experimental and observational constraints on the axion through the axion-photon coupling, with two prototypical axion models (KSVZ and DFSZ) and the nominally open mass range. Although somewhat schematic, it provokes several key points

The first concerns axion models. While it is encouraging that two very different axion models have axion-photon couplings of the same order of magnitude, one should be cautious in drawing conclusions. Should the ratio of the electromagnetic and color anomaly $E / N$ be far away from that corresponding to exact suppression, $(\sim 195)$, there could be a pleasant surprise for experimentalists, conversely a value close to it could make the axion nearly impossible to see. Kim has evaluated numerous models with very different but plausible PQ charges, [71] and nevertheless finds their corresponding $g_{A \gamma \gamma}{ }^{2}$ clustered in roughly two orders of magnitude, mostly more favorable than the implementation of the DFSZ model indicated throughout this review Second, the open range of axion masses $\left(m_{A}=10^{-(6-2)} \mathrm{eV}\right)$ is "soft" on both ends, particularly 
on the low-mass side. Taken together, a good point of view on these experiments is that they are principally discovery tools, a negative result is less meaningful even if the mass-coupling region covered is substantial

Regarding the experiments, it is also interesting that the axion-photon coupling $g_{A \gamma \gamma}$ has lent itself to the greatest variety of imaginative and practical search techniques. If the axion is someday found, it will likely be through the $\vec{E} \quad \vec{B}$ interaction. Purely laboratory experiments, such as the precision measurement of vacuum birefringence using lasers and magnets, are elegant and will soon provide beautiful demonstrations of higher-order QED. On the other hand, we do not see how any extrapolation of those techniques could ever be sensitive to DFSZ axions, although verifying the limits from HB stars by purely terrestrial experiments would be an important and significant achievement.

The most encouraging developments in the past two years has been the demonstration that the standard axion models could be reached with the microwave cavity experiments. Upgrades to extend their sensitivity and mass reach are already being planned In the meantime it is very important for those experiments to run as long as they can, the $(1-10 \mu \mathrm{eV})$ region of mass is arguably the most interesting range with which to begin.

The microwave cavity experiments are also not without limitations The local CDM density of our halo is not well-determined Furthermore, we do not see how to easily extend the mass search of the microwave cavity searches to cover the entire range, particularly upward. Individual cavities of larger diameter with arrays of metallic posts are a useful approach to higher frequencies, [49] as opposed to power-combining an unwieldy number of small cavities, each with its own tuning mechanism,[72] but this will require significant development. If $m_{A}>>10^{-3} \mathrm{eV}$, another technique will need to be found. It is impressive to consider that as small as the axion signal is expected to be-requiring radio receivers orders of magnitude more sensitive than anything else on earth--power sensitivity is not likely to be a limitation of the microwave cavity experiments, in view of the very promising work on DC SQUID amplifiers and the Rydberg atom single-quantum receiver.

Our many colleagues who assisted us in this review are unfortunately too numerous to acknowledge by name, but we are sincerely grateful for their help This work was supported by the US Department of Energy under Contract Nos. W-7405-ENG-048, DE-FC02-94ER40818 and National Science Foundation Grant no. PHY-9501959

*by Laurence Livermore National Laboratory 


\section{References}

[1] R Peccei and H Quinn, Phys Rev Lett 38 (1977) 1440.

[2] S Weinberg, Phys. Rev Lett 40 (1978) 223

[3] F. Wilczek, Phys Rev Lett 40 (1978) 279

[4] J E Kim, Phys. Rep 150 (1987) 1.

[5] P Sikivie, Phys Rev Lett 51 (1983) 1415; 52 (1984) 695(E); Phys Rev D32 (1985) 2988

[6] S DePanfilis et al, Phys Rev Lett. 59 (1987) 839, W Wuensch et al, Phys. Rev D40 (1989) 3153

[7] C Hagmann et al., Phys Rev D42 (1990) 1297

[8] C. Hagmann et al., Phys Rev Lett 80 (1998) 2043

[9] I Ogawa et al, Proc. of the 2nd RESCEU International Symposium on "Dark Matter in the Universe and its Direct Detection," Universal Academy Press, Inc (Tokyo), M. Minowa ed (1997)

[10] Michael S Turner, Phys Rep 197 (1990) 167

[11] G G. Raffelt, Phys. Rep 198 (1990) 1

[12] see, e.g, V Baluni Phys. Rev D19 (1979) 2227, R J Crewther et al., Phys Lett B88 (1979) 123

[13] IS Altarev et al., Yad Fiz 59 (1996) 1204 [Sov J. Nucl Phys 59 (1996) 1152].

[14] H.-Y Cheng, Phys. Rep 158 (1988) 1

[15] Ta-pei Cheng and Ling-Fong Li, Gauge Theory of Elementary Particle Physics, Oxford Univ Press, New York, (1984) pp. 492-3

[16] In this model, the broken chiral U(1) symmetry arises from a new heavy weakinteraction quark and a weak-interaction zero weak-hypercharge singlet. $c f$, J E Kim, Phys Rev Lett 43 (1979) 103; M.A Shifman, A I Vainshtein and V I. Zakharov, Nucl. Phys. B166 (1980) 493

[17] In this model, the broken chiral $\mathrm{U}(1)$ symmetry arises from a new $\mathrm{SU}(2) \times \mathrm{U}(1)$ singlet complex scalar field $\phi$ One of the scalar doublets, of hypercharge -1 , couples to right-handed charge $+2 / 3$ quarks. The other scalar doublet, of hypercharge +1 , couples to right-handed charge $-1 / 3$ quarks and right-handed charged leptons $c f, \mathrm{M}$ Dine, W Fischler, and M Srednicki, Phys Lett B104 (1981) 199, A R. Zhitnitsky, Yad Fiz 31 (1980) 497 [Sov J Nucl. Phys 31 (1980) 260]

[18] T W Donnelly et al, Phys Rev D18 (1978) 1607 
[19] F Reines, H S Gurr, and H W Sobel, Phys Rev Lett 37 (1976) 315

[20] H S Gurr, F Reines, and H W. Sobel, Phys Rev Lett. 33 (1974) 179

[21] A F Rothenberg, thesis RX-1311, Stanford University, Dept of Physics (May 1972), also distributed as Stanford Linear Accelerator report SLAC-0147 (May 1972) Experiment details may be found in SLAC-PROPOSAL-E-056 (Jan 1970)

[22] P Bossetta et al, Phys Lett. B74 (1978) 143

[23] P Alibran et al, Phys. Lett B74 (1978) 134, this was a reported excess of $\nu_{e}$ and $\bar{\nu}_{e}$ events.

[24] T Hansl et al, Phys Lett B74 (1978) 139

[25] These peaks may have be an artifact, $c f, \mathrm{R}$ Betts et al, "Positron Production in Heavy Ion Collisions: Current Status of the Problem," Nucl Phys A583 (1995) 247c

[26] E.M Riordan et al., Phys. Rev Lett. 59 (1987) 755.

[27] J D. Bjorken et al , Phys Rev D38 (1988) 3375

[28] M Sivertz et al., Phys Rev D26 (1982) 717.

[29] M S Alam et al., Phys. Rev D27 (1983) 1665.

[30] C. Edwards et al, Phys Rev. Lett. 48 (1982) 903

[31] B Niczyporuk et al, $Z$ Phys C17 (1983) 197

[32] G Mageras et al., Phys Rev Lett 56 (1986) 2672

[33] G. G Raffelt, Stars as Laboratories for Fundamental Physics, University of Chicago Press, Chicago (1996)

[34] J A Frieman, S Dimopoulos and Michael S. Turner, Phys. Rev D36 (1987) 2201

[35] G.G. Raffelt and D S P Dearborn, Phys. Rev D36 (1987) 2211.

[36] D S P Dearborn, D N Schramm and G Steigman, Phys Rev. Lett 56 (1986) 26

[37] R P Brinkmann and Michael S Turner, Phys. Rev D38 (1988) 2338

[38] A Burrows, Michael S. Turner and R P Brinkmann, Phys Rev D39 (1989) 1020

[39] Michael S Turner, Phys Rev Lett 60 (1988) 1797

[40] J Preskill, M Wise and F Wilczek, Phys Lett 120B (1983) 127, L Abbott and $\mathrm{P}$ Sikivie, ibid, 133, $\mathrm{M}$ Dine and $\mathrm{W}$ Fischler, ibid, 137 
[41] An insightful, if somewhat fanciful description of the axion may be found in an article "A Pool-Table Analogy to Axion Physics", P Sikivie, Physics Today 49 (1996) 22

[42] Michael S Turner, Phys Rev D33 (1986) 889

[43] A Vilenkin and E.PS Shellard, "Cosmic Strings and Other Topological Defects," Cambridge Univ Press (1994)

[44] R. Davis, Phys Lett 180B (1986) 225

[45] A complete historical list of references would be unproductive For the most recent status, see R A. Battye and E P S Shellard, Phys Rev Lett 73 (1994) 2954, and erratum ibid., 76 (1996) 2203, R A Battye and E P S Shellard, Nucl Phys B423 (1994) 260 on the one hand, and C. Hagmann and P Sikivie, Nucl. Phys B363 (1991) 247 on the other

[46] Michael S Turner, Phys. Rev D42 (1990) 3572.

[47] P. Sikivie and J R. Ipser, Phys Lett B291 (1992) 288

[48] P Sikivie, I.I Tkachev and Y Wang, Phys Rev Lett. 75 (1995) 2911

[49] C Hagmann et al., Rev. Sci Instrum, 61 (1990) 1076

[50] E J Gates, G Gyuk and Michael S Turner, Astrophys. J 449 (1995) 123.

[51] J Clarke, in The New Superconducting Electronics, H. Weinstock and R W. Ralston, eds., Kluwer Academic Publishers (1993) 123.

[52] M Mück et al, Appl. Phys Lett. 72 (1998) 2885

[53] T. F Gallagher, Rydberg Atoms, Cambridge University Press, Cambridge (1994).

[54] D. Kleppner and T.W Ducas, Bull. Am Phys Soc. 21 (1976) 200

[55] S. Matsuki, private communication (1998)

[56] G.G Raffelt and L Stodolsky, Phys Rev D37 (1988) 1237

[57] K van Bibber, N Dagdeviren, S.E Koonin, A K. Kerman, and H N Nelson, Phys Rev Lett 59 (1987) 759.

[58] G Ruoso et al, Z Phys C56 (1992) 505

[59] L. Maiani, R Petronzio and E Zavattini, Phys Lett B175 (1986) 359

[60] Y Semertzidis et al, Phys Rev Lett 64 (1990) 2988, R. Cameron et al, Phys Rev. D47 (1993) 3707

[61] S A Lee et al, FNAL proposal E-877 (1995)

[62] M Bershady et al, Phys Rev Lett 66 (1991) 1398

[63] K van Bibber et al, Phys Rev D39 (1989) 2089 
[64] D M Lazarus et al, Phys Rev Lett 69 (1992) 2333

[65] M Minowa et al., in Proc NANP-97-Int'l Workshop on Non-Accelerator New Physics, Dubna, July 7-11 (1997)

[66] F T Avignone III et al, in Proc NANP-97-Int'l Workshop on Non-Accelerator New Physics, Dubna, July 7-11 (1997)

[67] J E Moody and F Wilczek, Phys Rev D30 (1984) 130

[68] W -T Ni et al, in Proc. 7th Marcel Grossmann Meeting on General Relativity, World Scientific (1996) 1625, W-T Ni, Class Quant. Grav 13 (1986) A135.

[69] A N Youdin et al., Phys Rev Lett, 77 (1996) 2170

[70] Joshua C Long, Hilton W Chan, John C Price, University of Colorado, Boulder, preprint COLO-CMP-9805-011 (May, 1998) and e-print archive hepph/9805217; John Price, "Search for New Gravitational Strength Forces", Univ of Colorado, Boulder, proposal to the National Science Foundation (1996); Ho Paik, "Search for Axion-Like Particles Using a Superconducting Accelerometer," Univ of Maryland, College Park, proposal to the National Science Foundation (1997)

[71] J E Kim, to be published in Proceedings of the Second International Workshop on Axions, University of Florida, March 13-15, 1997

[72] C Hagmann, Thesis, University of Florida, Dept. of Physics (1990) 
Fig 1 Limits on axion lifetime versus axion mass in the $A \rightarrow \gamma \gamma$ decay channel Also shown is the lifetime-mass relation for a usual weak-scale axion (From Ref [27])

Fig 2 Summary of the limits on the axion mass from stellar evolution and cosmology (From Ref [33])

Fig 3 The local phase space distribution $d f / d u$ for thermalized cosmic axions The horizontal axis is the axion kinetic energy relative to its mean The tallest spectrum is the distribution in the galactic rest frame. The other two curves display the expected axion distribution at the earth's frame, as measured in December (next-tallest), and in June (shortest). (From Ref [46])

Fig 4 The velocity spectrum of axions at our solar system, predicted in the model of Refs. [47,48] (Upper panel) No initial angular momentum (Lower panel) Finite initial angular momentum Scattering processes are expected to eventually thermalize the spectrum, leading to the lower energy lines being subsumed into a Maxwellian-like distribution of width $\Delta E_{A} / E_{A} \sim 10^{-(6-7)}$

Fig. 5 Schematic of the Rochester-Brookhaven-Fermilab microwave cavity experiment to search for cosmic axions. (From Ref [6].)

Fig 6 Exclusion region for present and future microwave cavity experiments. Note the plot is flattened by presenting $\left(g_{A \gamma \gamma} / m_{A}\right)^{2}$ Shown are the experiments of Rochester-BNL-FNAL, [6] the University of Florida, [7] the US large-scale experiment,[8] and the Kyoto experiment.[9] The sensitivity possible for the large-scale experiment upgraded with DC SQUID amplifiers is indicated by the shaded dashed line All are normalized to $90 \% \mathrm{cl}$. and $\rho_{\mathrm{CDM}}=7.5 \times 10^{-25} \mathrm{~g} / \mathrm{cm}^{3}$.

Fig 7. Cutaway diagram of the large-scale U.S. axion search

Fig 8 Schematic of the receiver for the large-scale U S axion experiment. Note the two data streams, one medium resolution (125 Hz/channel, $\Delta E / E \sim \mathcal{O}\left(10^{-7}\right)$ ), and one high resolution $\left(0.02 \mathrm{~Hz} /\right.$ channel, $\left.\Delta E / E \sim \mathcal{O}\left(10^{-11}\right)\right)$.

Fig 9 Histogram of the dispersion of the combined single bin data, with power excess in units of the r.m.s. deviation about the mean power, for the large-scale U.S. axion search The smooth curve is the distribution based on Gaussian thermal noise treated as input to the analysis chain

Fig 10. For the large-scale US axion search (a) Detected power expected from KSVZ axions; (b) the noise background were the axion to be distributed over six channels, and (c) the noise background were the axion signal to be confined to one channel

Fig. 11 Schematic of a DC SQUID amplifier

Fig 12 Power gain of a prototype DC SQUID utilizing stripline coupling, for various numbers of input stripline turns (From Ref [52]) 
Fig 13 Noise temperature of a prototype DC SQUID amplifier utilizing stripline input coupling at physical temperatures of 42 and $18 \mathrm{~K}$, in the UHF signal frequency range The narrow structures are not intrinsic to the SQUID, but rather are due to shaping in post-amplification (Figure courtesy M Mück)

Fig 14 A sketch of the apparatus used in the Kyoto Axion Search experiment

Fig 15 Phenomena associated with light propagating in an external electromagnetic field (a) Production of a real axions (b) The production of virtual axions (c) Higher-order QED All can affect the polarization state of light In general, (a) will lead to depletion of the photon beam (and the creation of an axion beam), and a rotation of the plane of its polarization, (b) and (c) lead to birefringence of the vacuum, i $e$ linearly polarized light becoming elliptically polarized

Fig 16 (a) The photon regeneration experiment. (b) Homodyning which eliminates the dependence of the sensitivity of the experiment to any external noise source

Fig 17 Dashed curve Limit possible from the simple photon regeneration experiment with the benchmark values of Eqn. 12. Solid curve: Limit possible by using $30+30$ SSC dipole magnets $(16.6 \mathrm{~m}, 66 \mathrm{~T})$, and a high-power $\mathrm{CO}_{2}$ laser $(100 \mathrm{~kW}$ circulating power) Dot-dashed curve Same, but where all combinations of alternating magnet polarity are used.

Fig 18 (a) Rotation of polarization of light, due to the attenuation of the parallel component by production of axions (b) Elliptical polarization resulting from a phase shift between parallel and perpendicular components of the light, due to QED, axion-photon mixing, and magnetically-induced birefringence of any residual gas, $i$ e the Cotton-Mouton effect 
Fig. 19 Schematic view of the polarization experiment to look for pseudoscalars, and QED light-by-light scattering (a) The ellipsometer, (b) the optical cavity inside the superconducting dipole magnets

Fig 20 Exclusion region for the coupling of axions with two photons $\left(M=1 / g_{A \gamma \gamma}\right)$. (a) From the optical rotation data. (b) From ellipticity data

Fig 21 Spectrum of A2218 at approximately one core radius, and the "on-off field" subtraction spectrum (b) Synthetic line introduced into the spectrum of A2256, from the decay of a $32 \mathrm{eV}$ axion In both cases, the intensity is in units of $10^{-18} \mathrm{erg} \mathrm{cm}^{-2} \operatorname{arcsec}^{-2} \AA^{-1} \mathrm{~s}^{-1}$

Fig. 22 Calculated spectrum of solar axions

Fig 23 Concept for the solar axion experiment utilizing a solenoidal magnet The central volume would be filled with the dispersion-matching gas, either $\mathrm{H}_{2}$ or $\mathrm{He}$, and a cylindrical multiple-wire proportional chamber on the inner wall for detection of the x-rays.

Fig 24. Exclusion region (99 7\% c l) from the solar axion search of Ref. [64]. The curves represent operation at different gas pressures (a) 0 Torr, (b) 55 Torr, (c) 100 Torr

Fig. 25 Limits from searches for new macroscopic forces. The horizontal axis is the range of the new force (the region between the vertical lines corresponds to the allowed axion window) The vertical axis is the product of coupling constants The diagonal lines are the expected couplings should the residual strong CP violation parameter $\vec{\theta}$ be $10^{-9}$ (conservatively allowed by experiments) or $10^{-14}$ (suggested should weak CP violation arise from the Kobayashi-Maskawa model) The upper limits to the couplings shown are those of Refs $[68,69]$

Fig 26 Plot of excluded $g_{A \gamma \gamma}$ vs $m_{A}$ with all experimental and observational constraints, along with predicted couplings for KSVZ and DFSZ models 


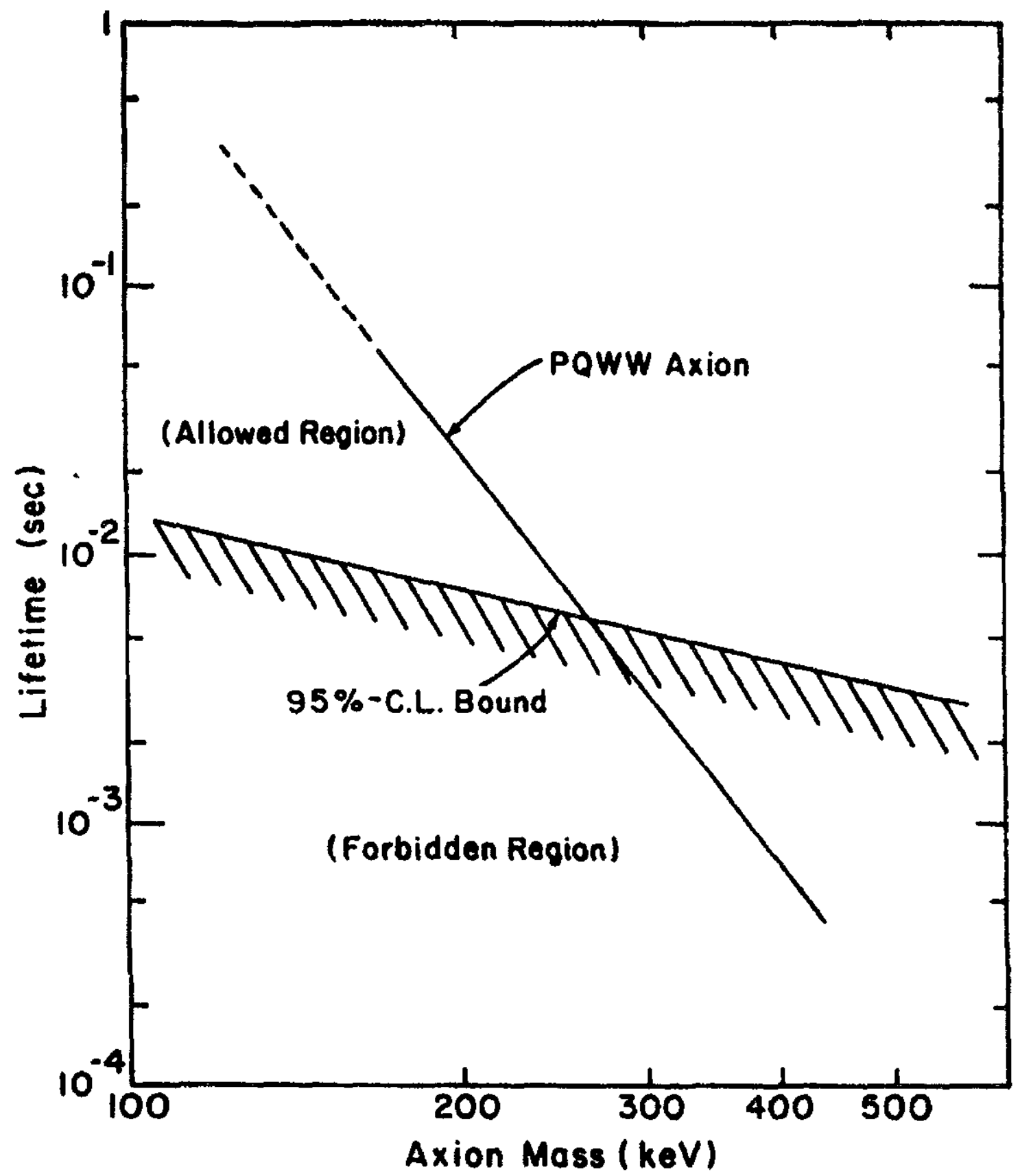

Figure 1 


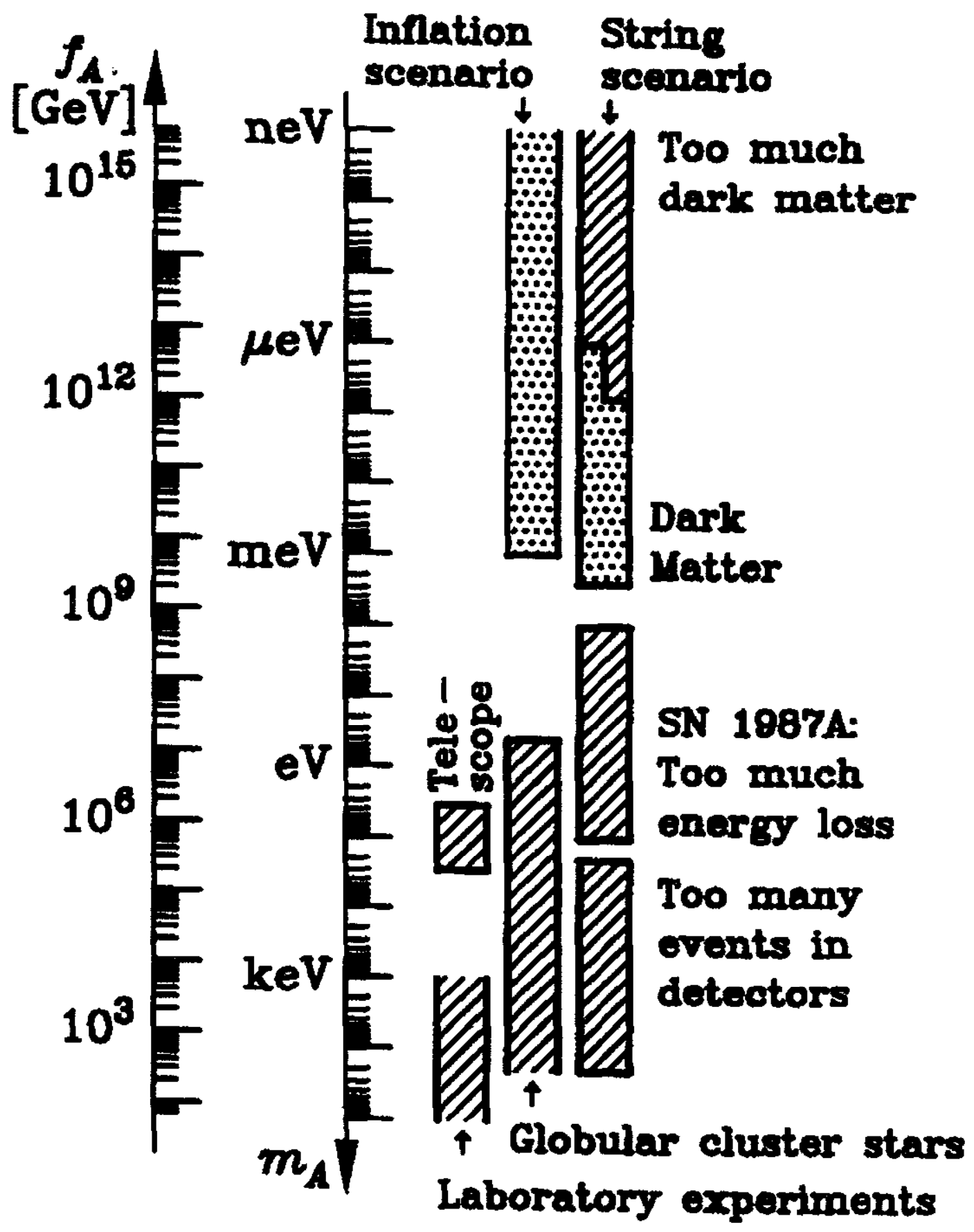

Figure 2 


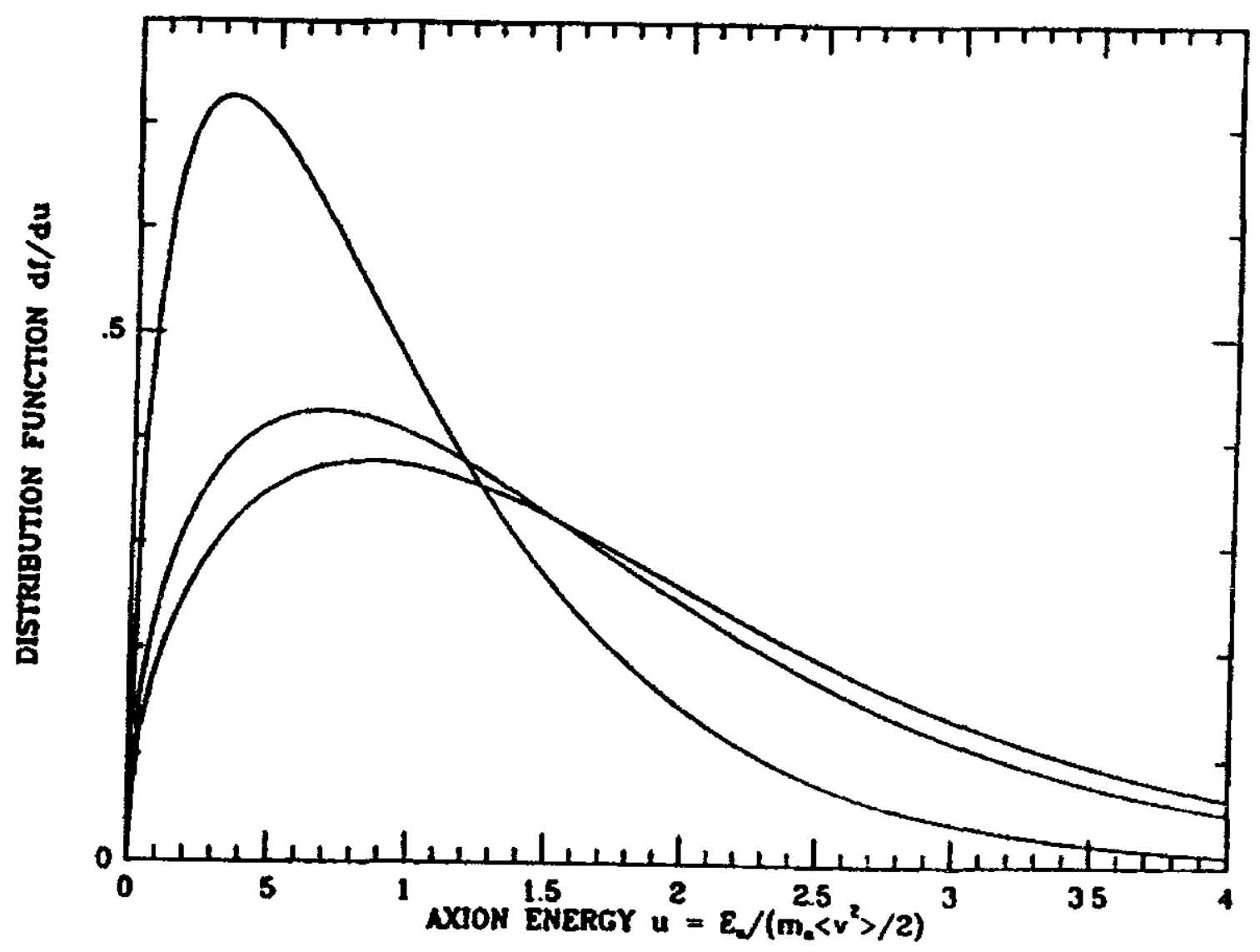

Figure 3 


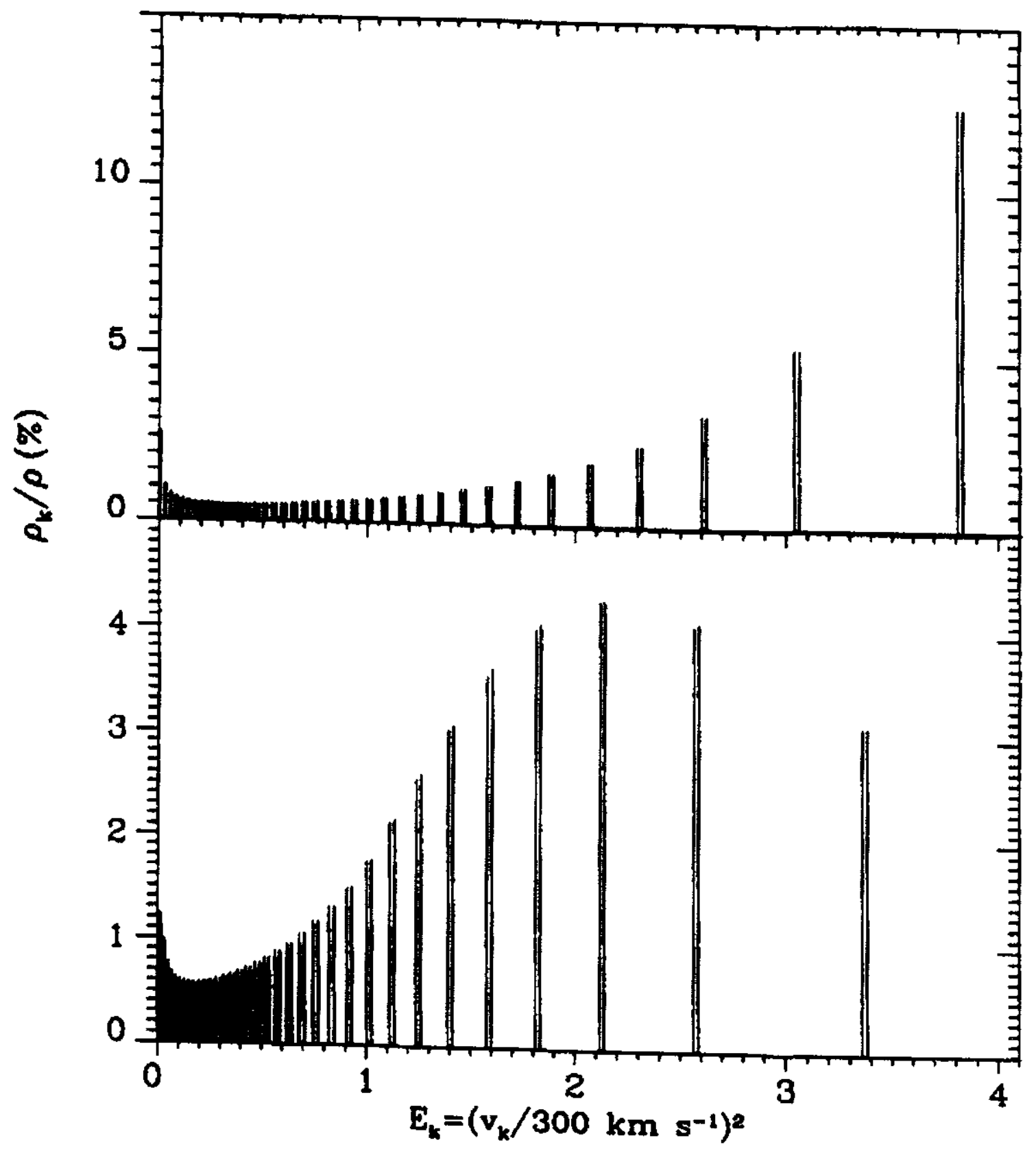

Figure 4 


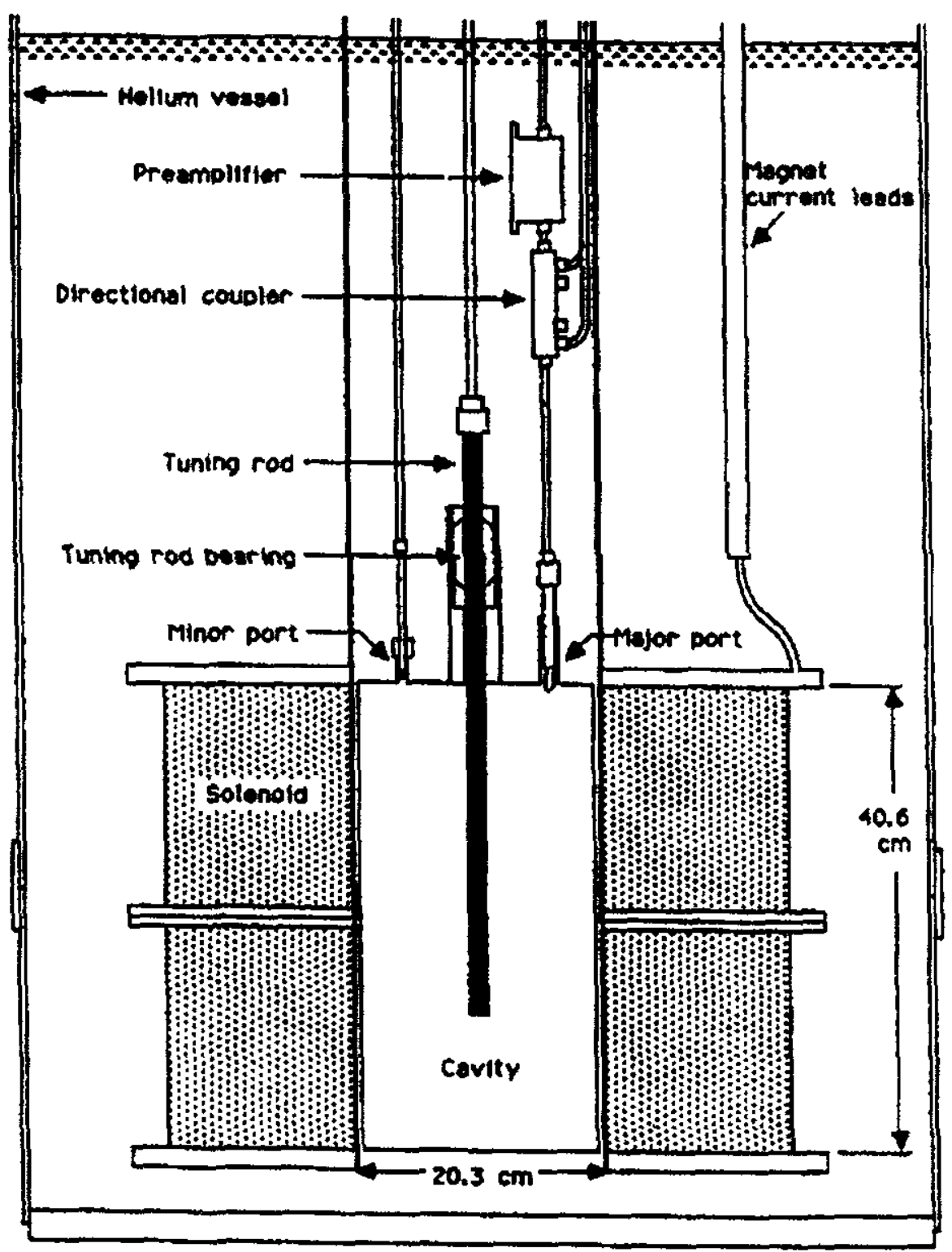

Figure 5 


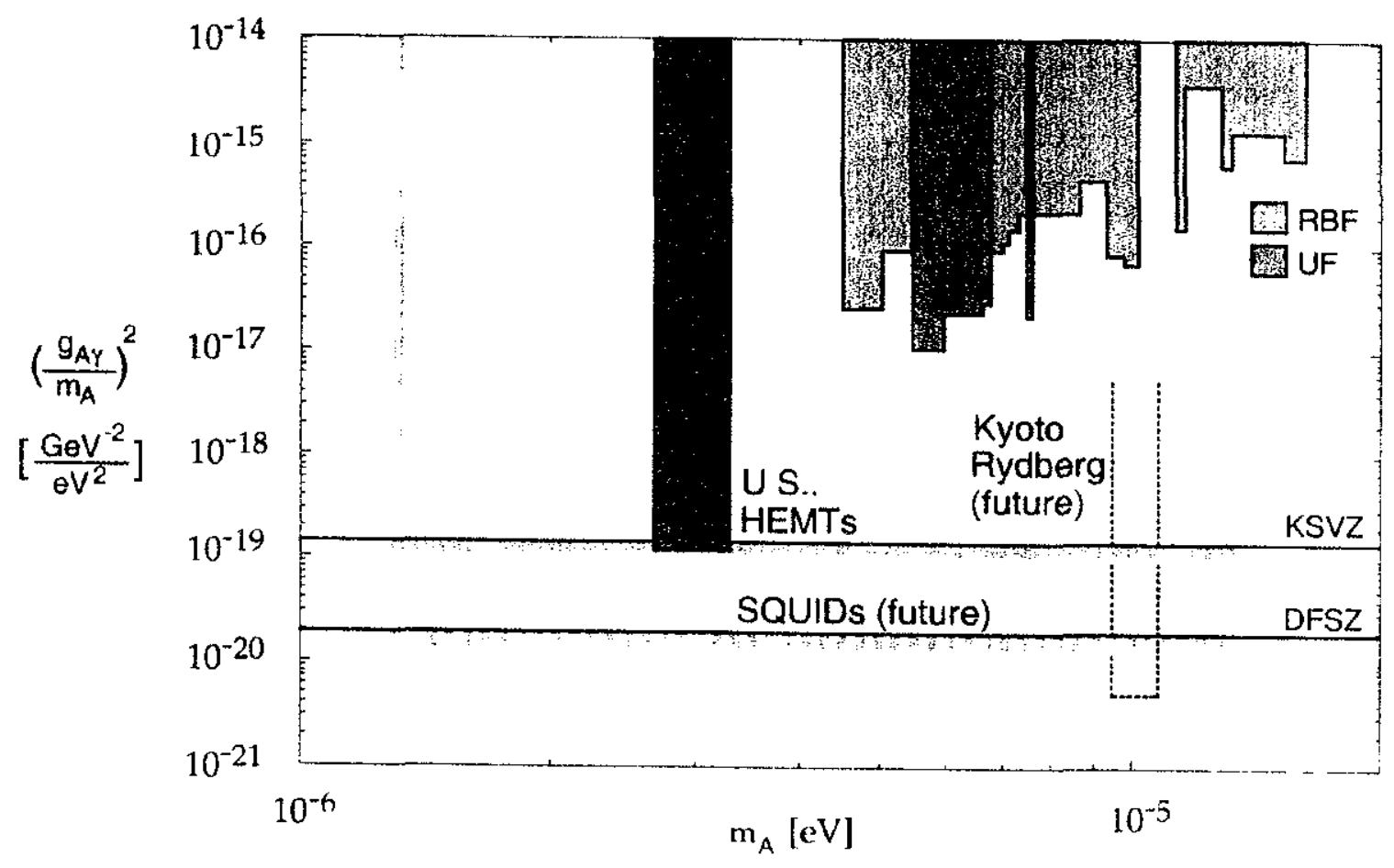

Figure 6 


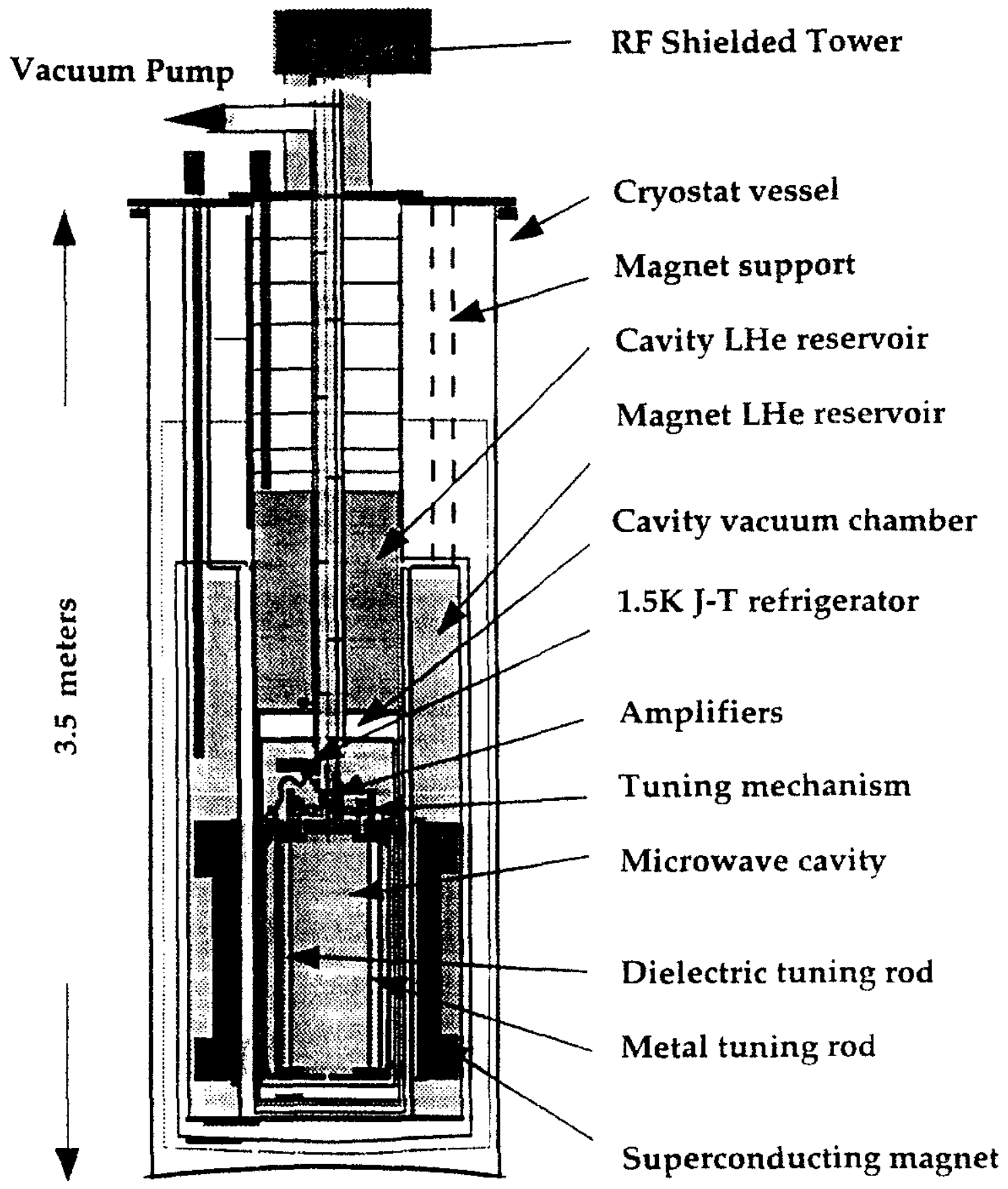

Figure 7 


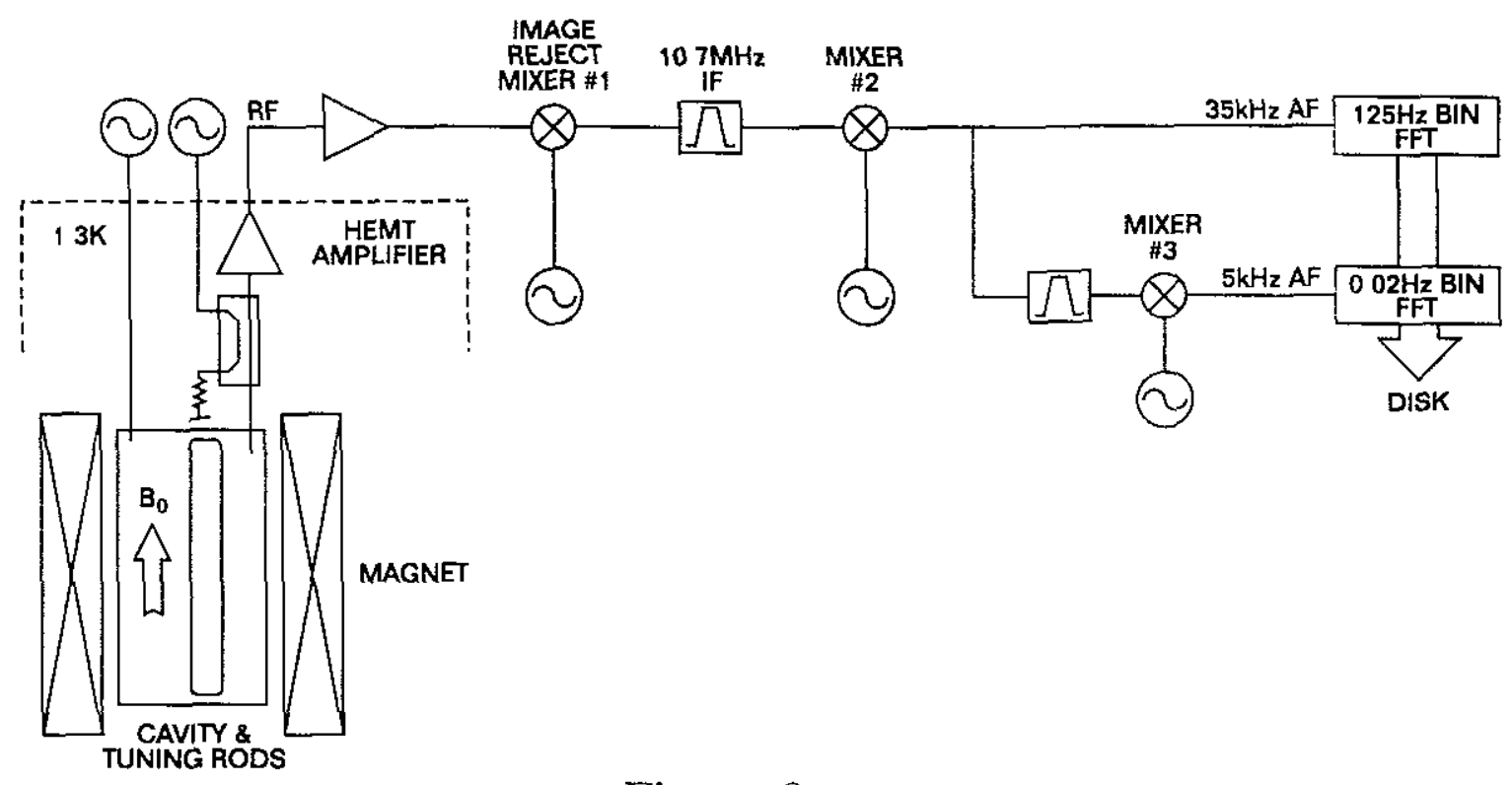

Figure 8

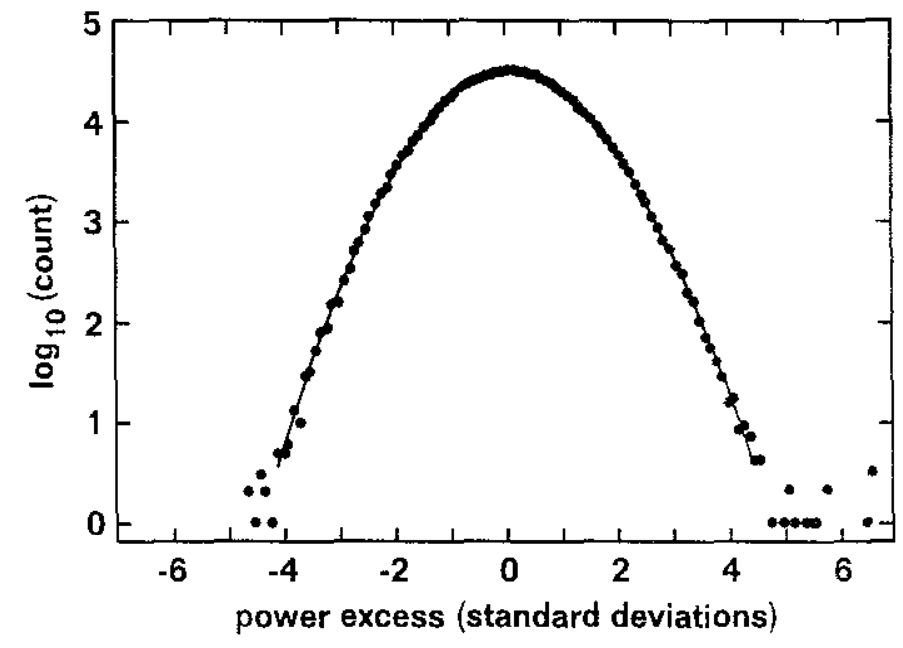

Figure 9 


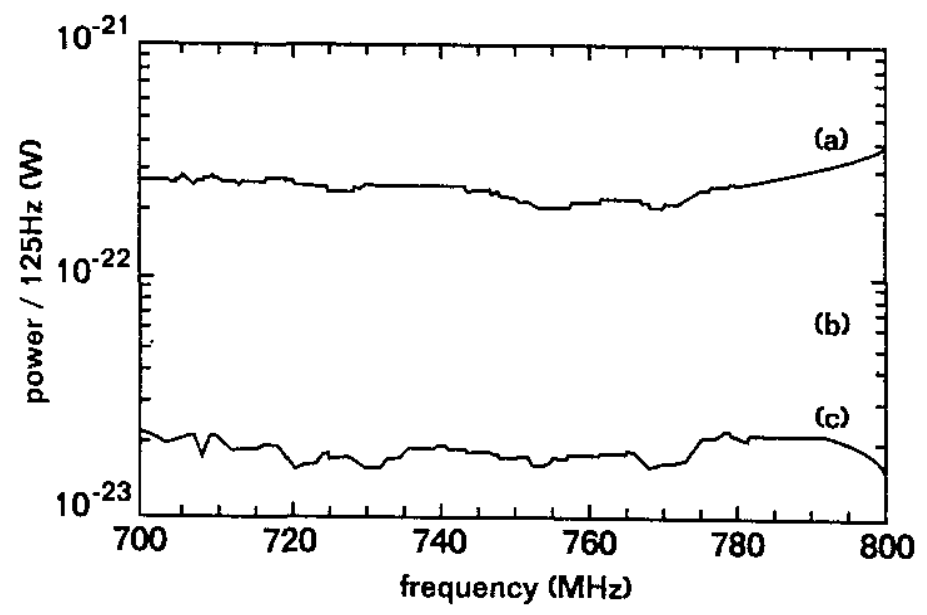

Figure 10
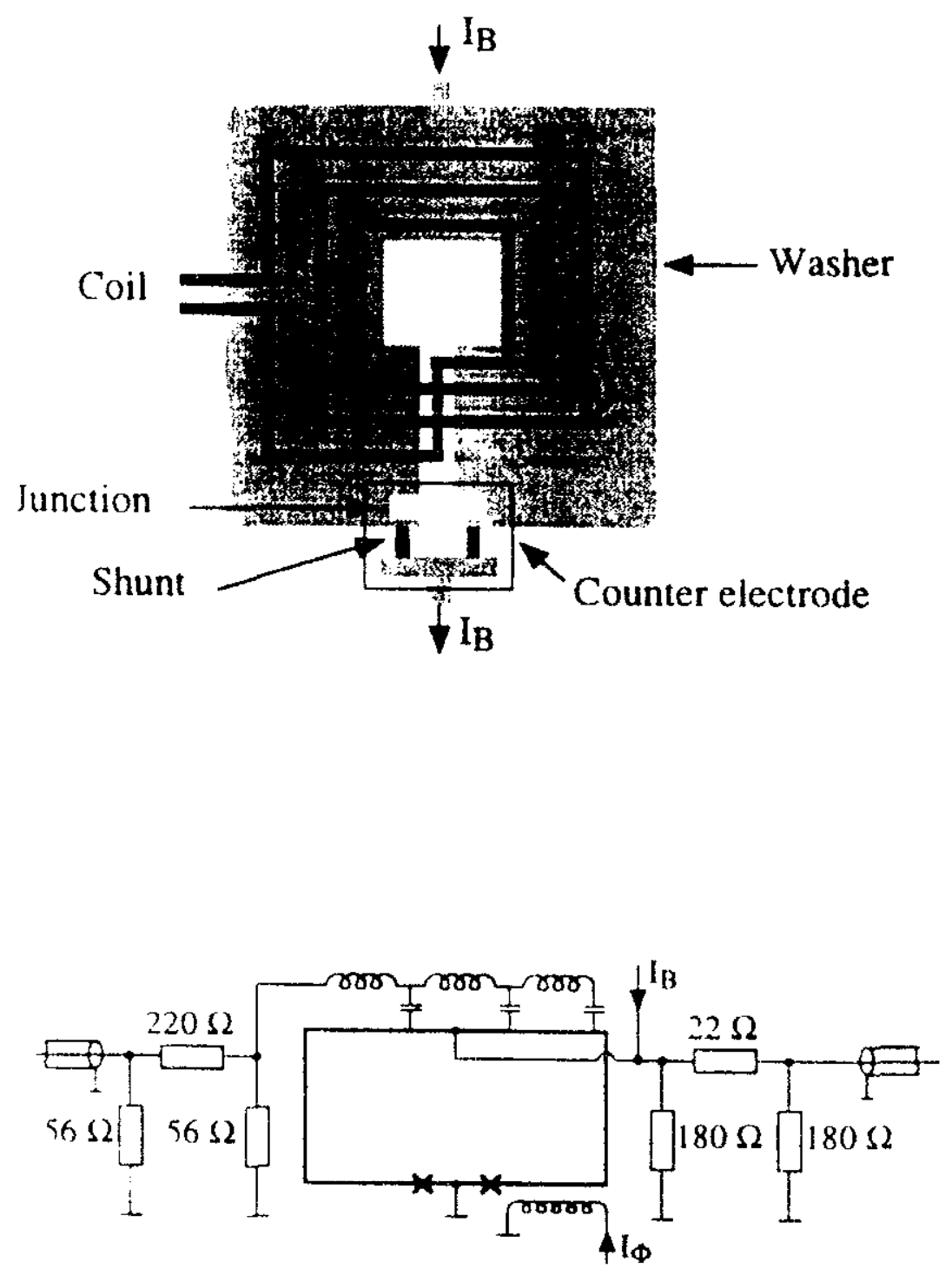

Figure 11 


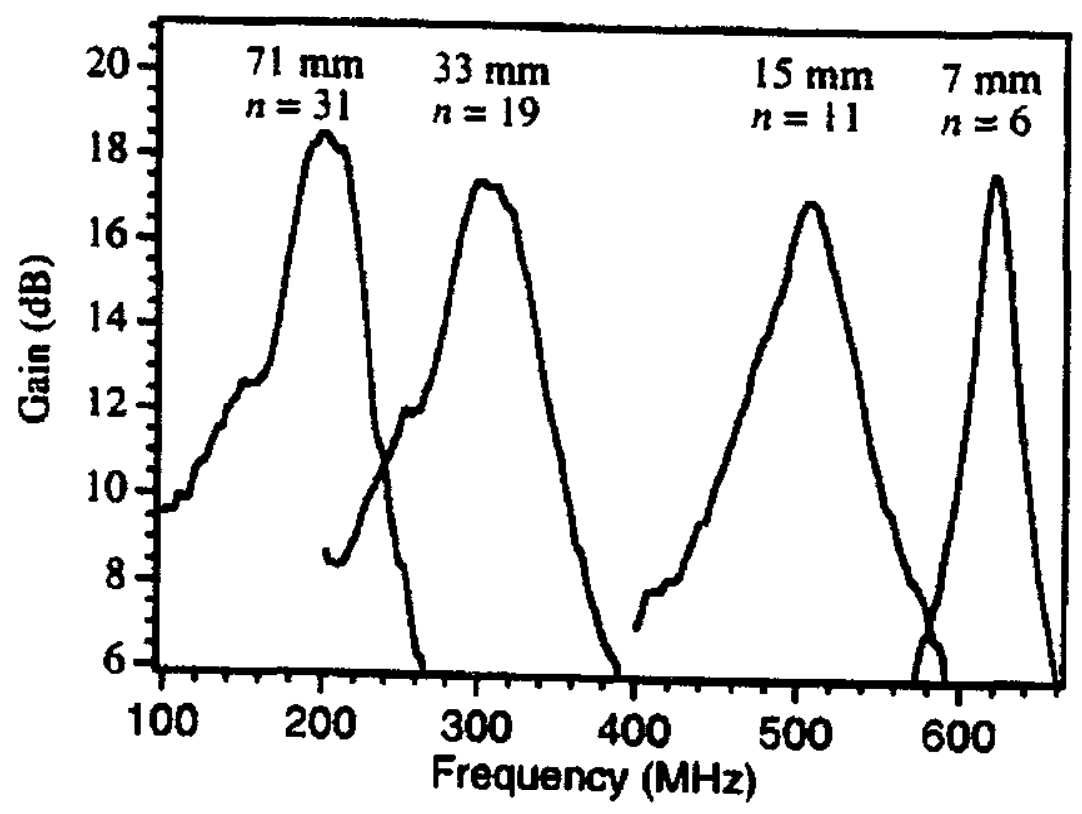

Figure 12

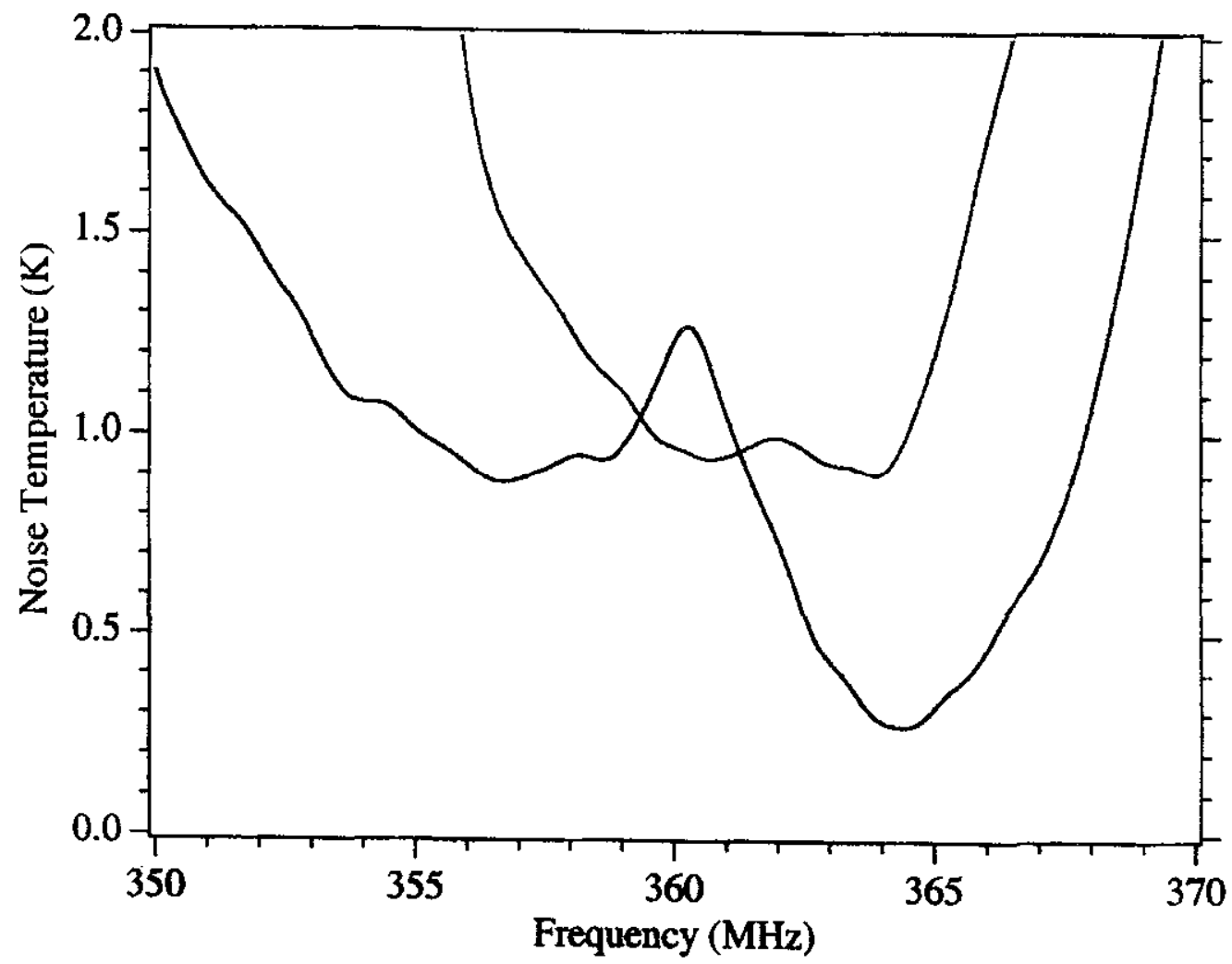

Figure 13 


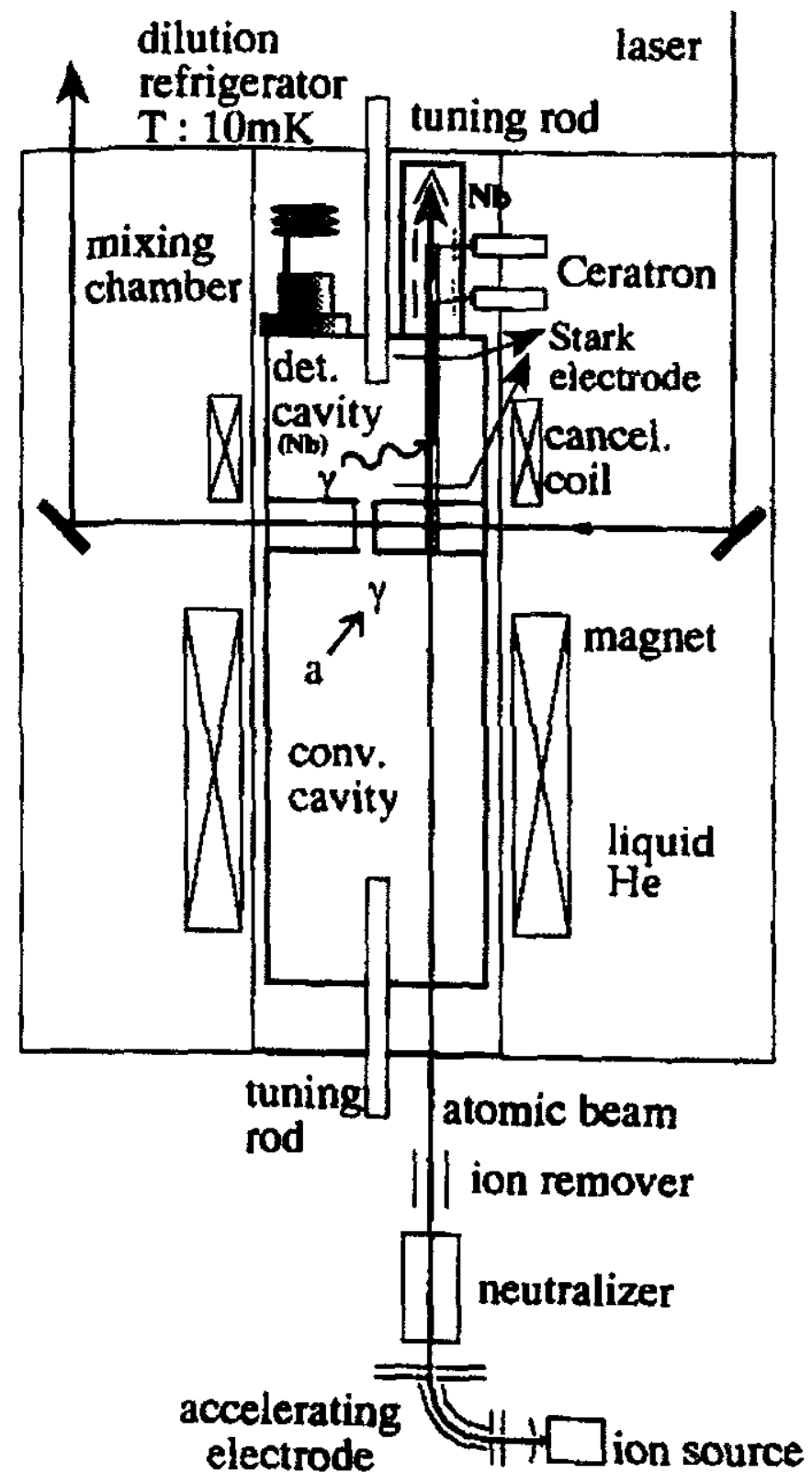

Figure 14 

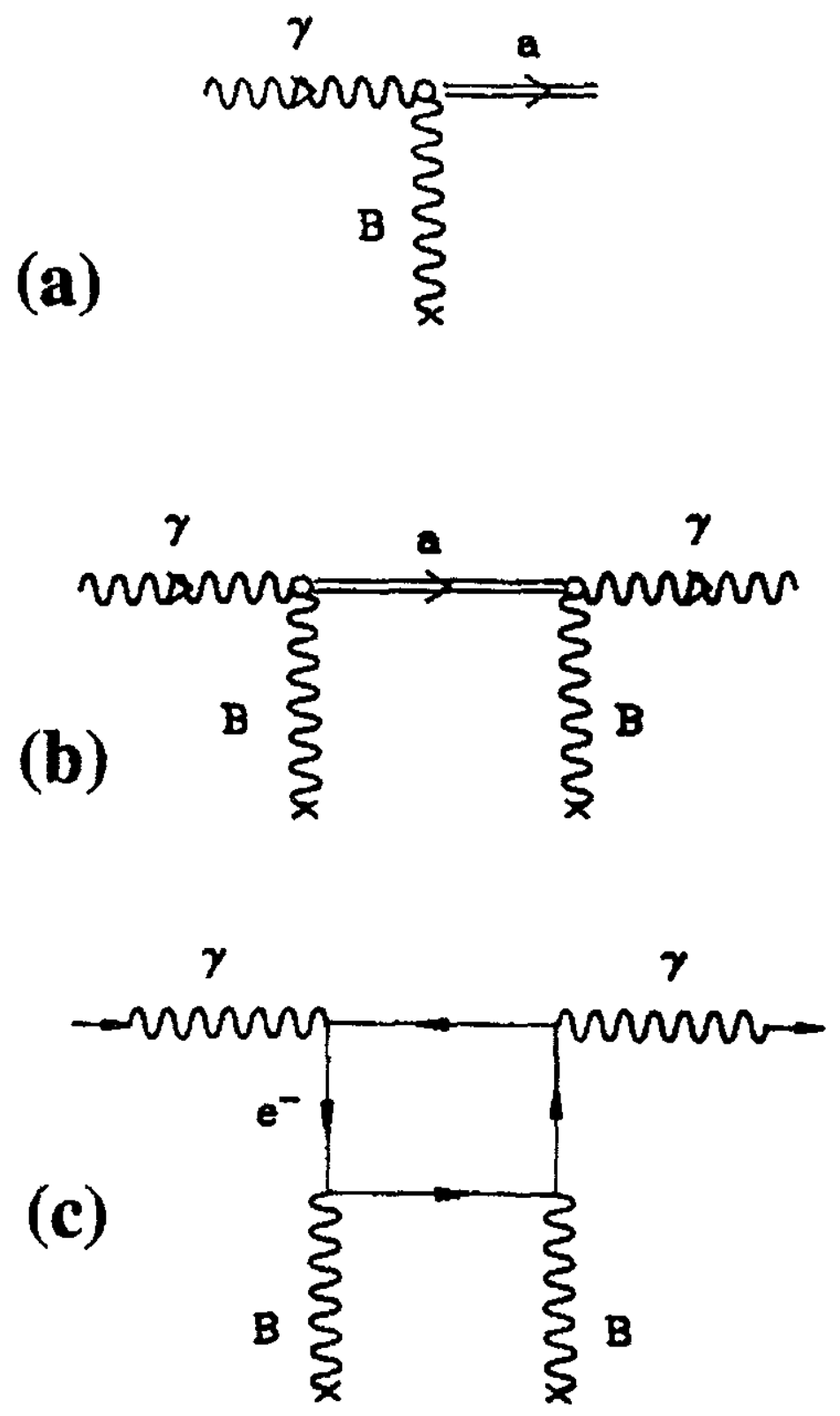

Figure 15 
(a)

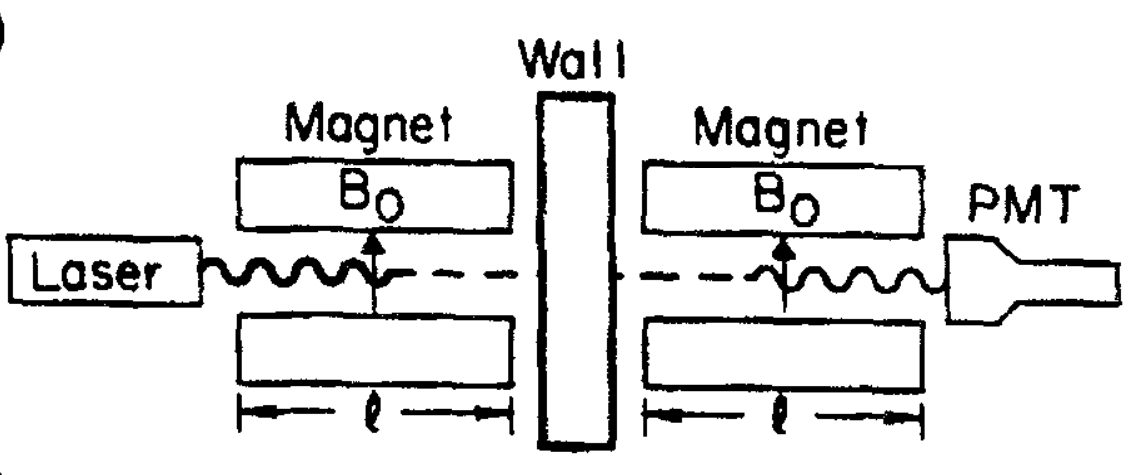

(b)

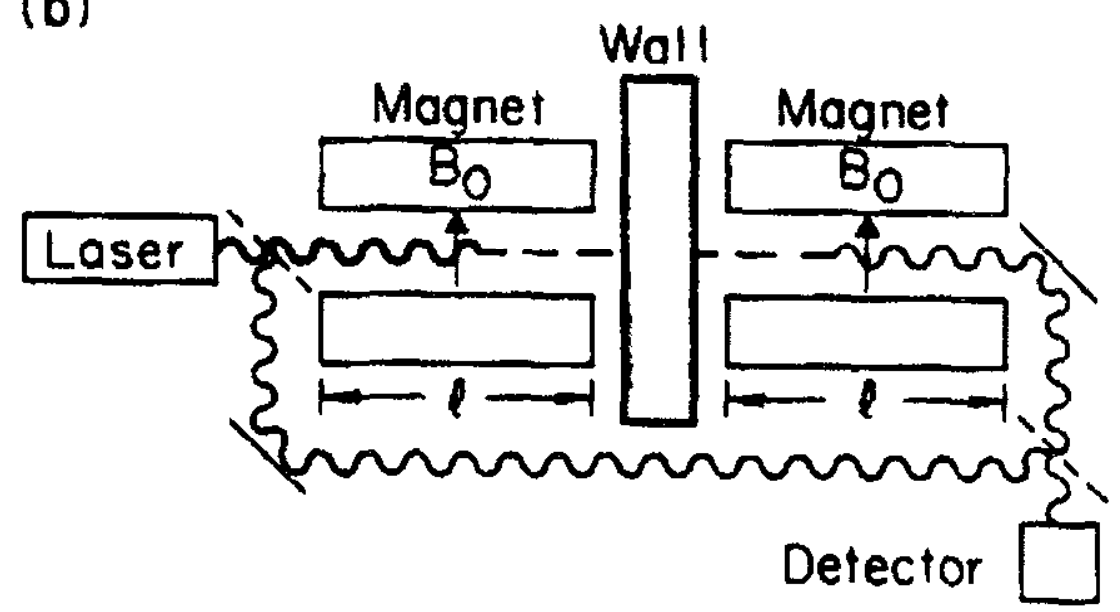

Figure 16

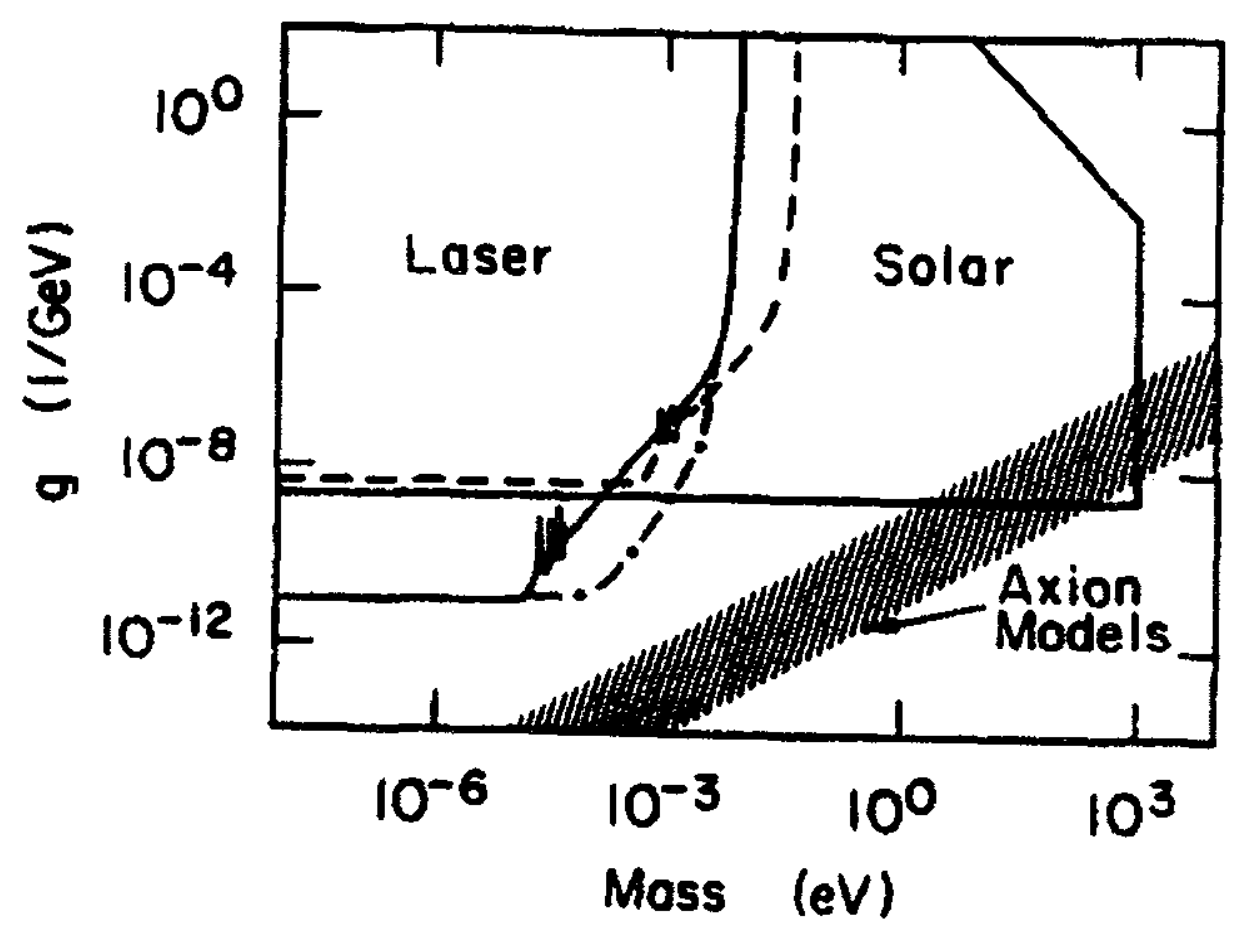

Figure 17 

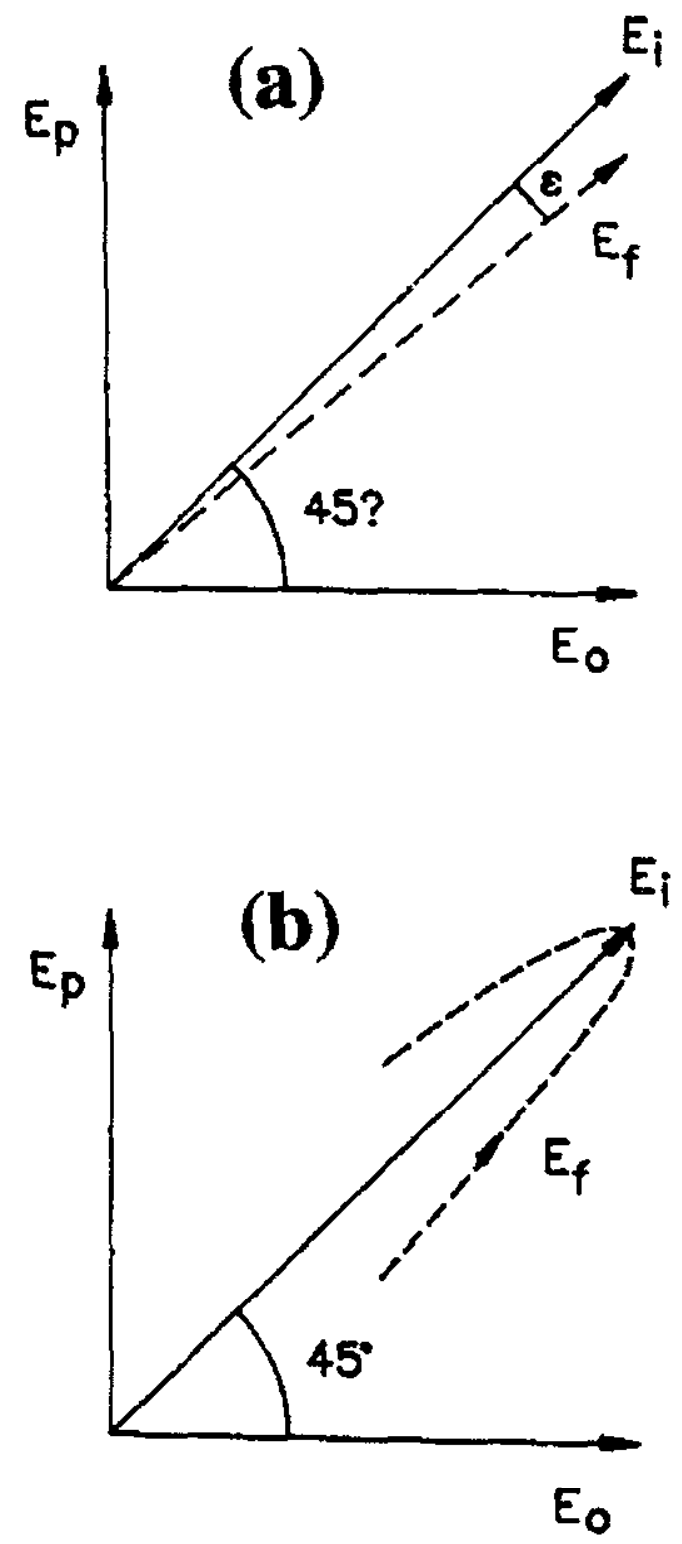

Figure 18 


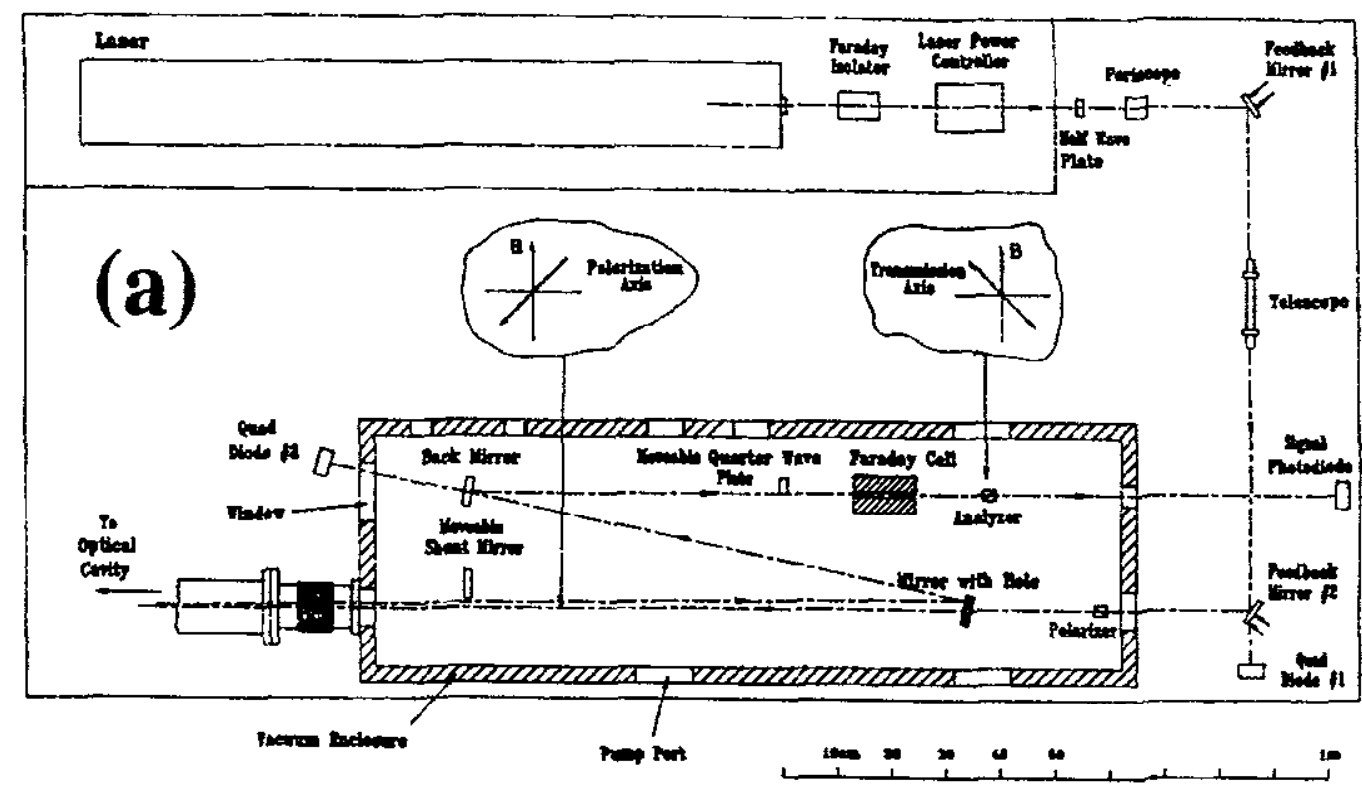

(b)

ENTRANCE MIRROR

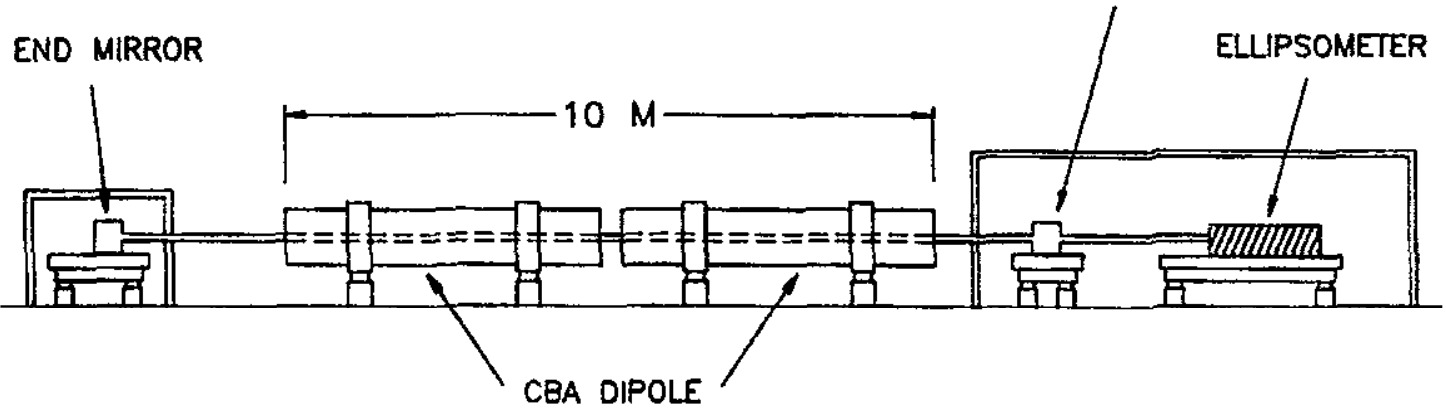

Figure 19 

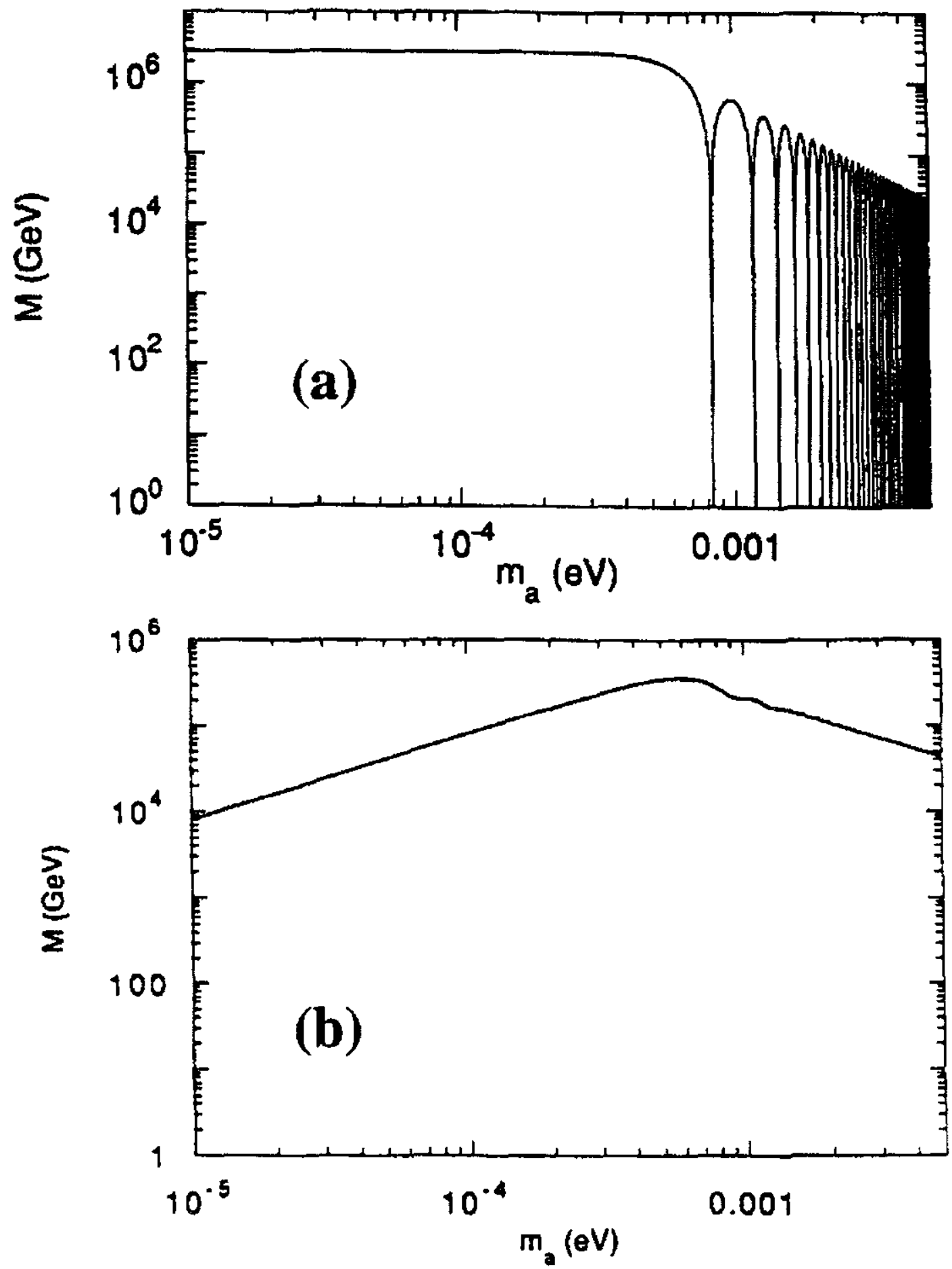

Figure 20 

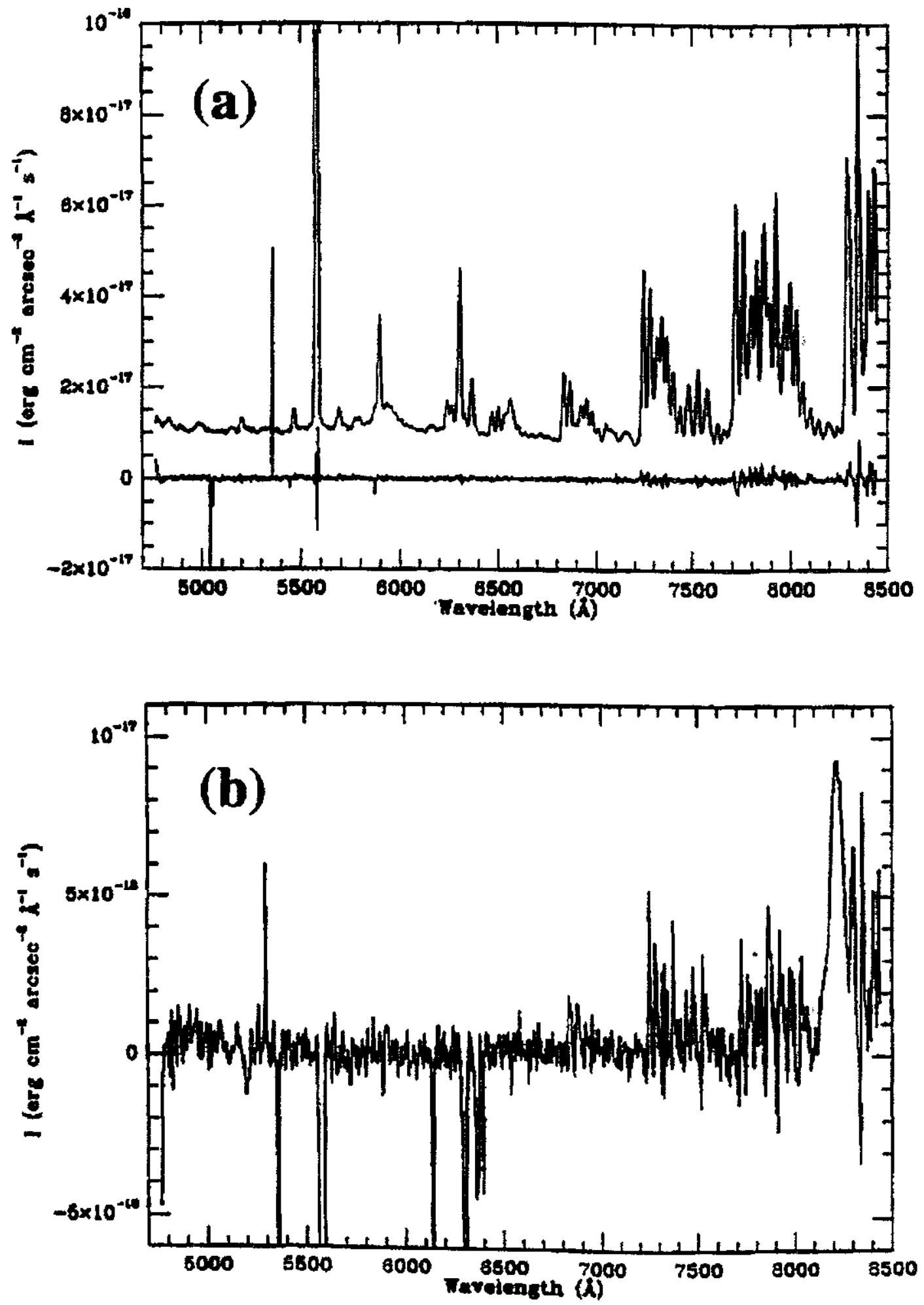

Figure 21 


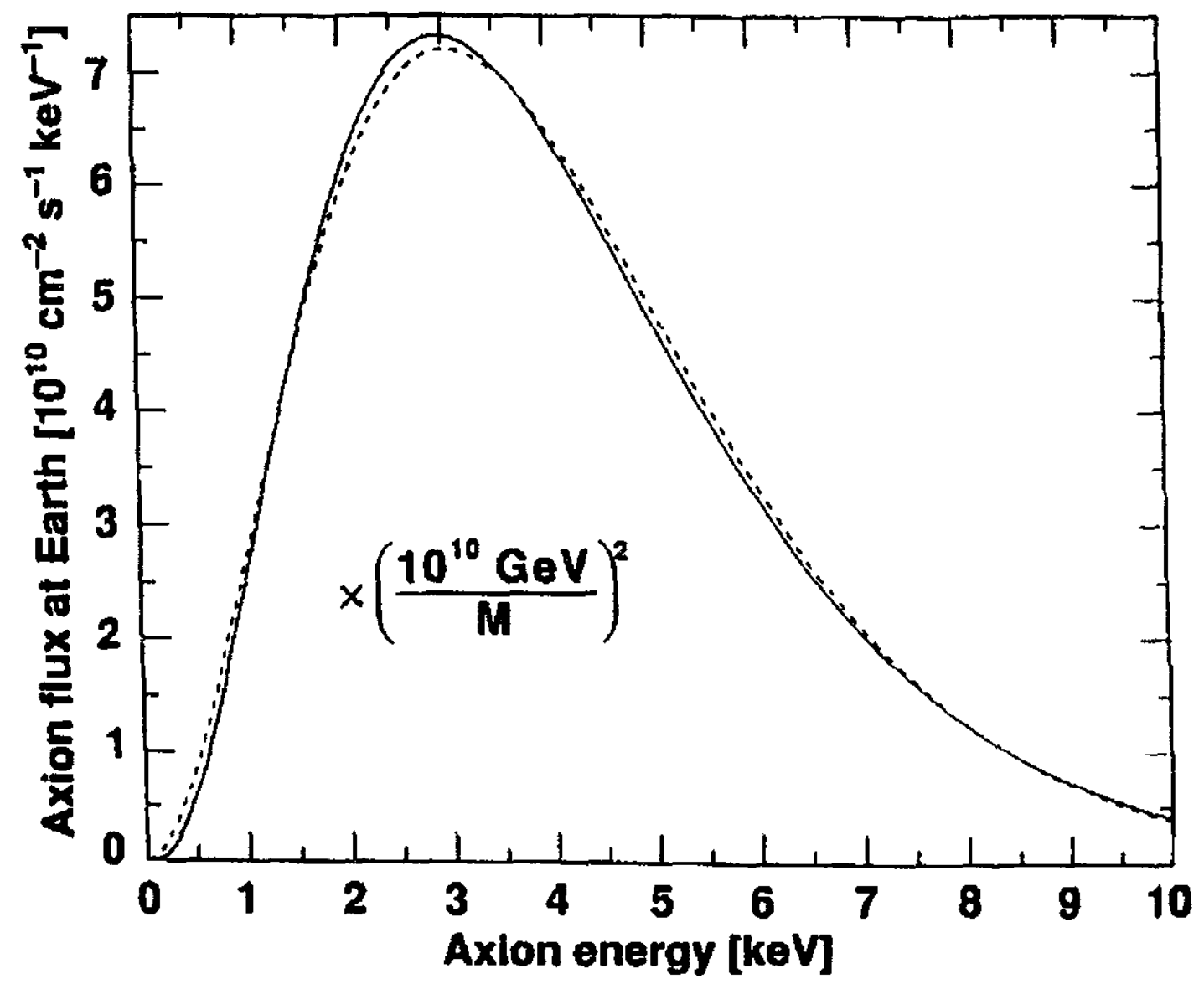

Figure 22 


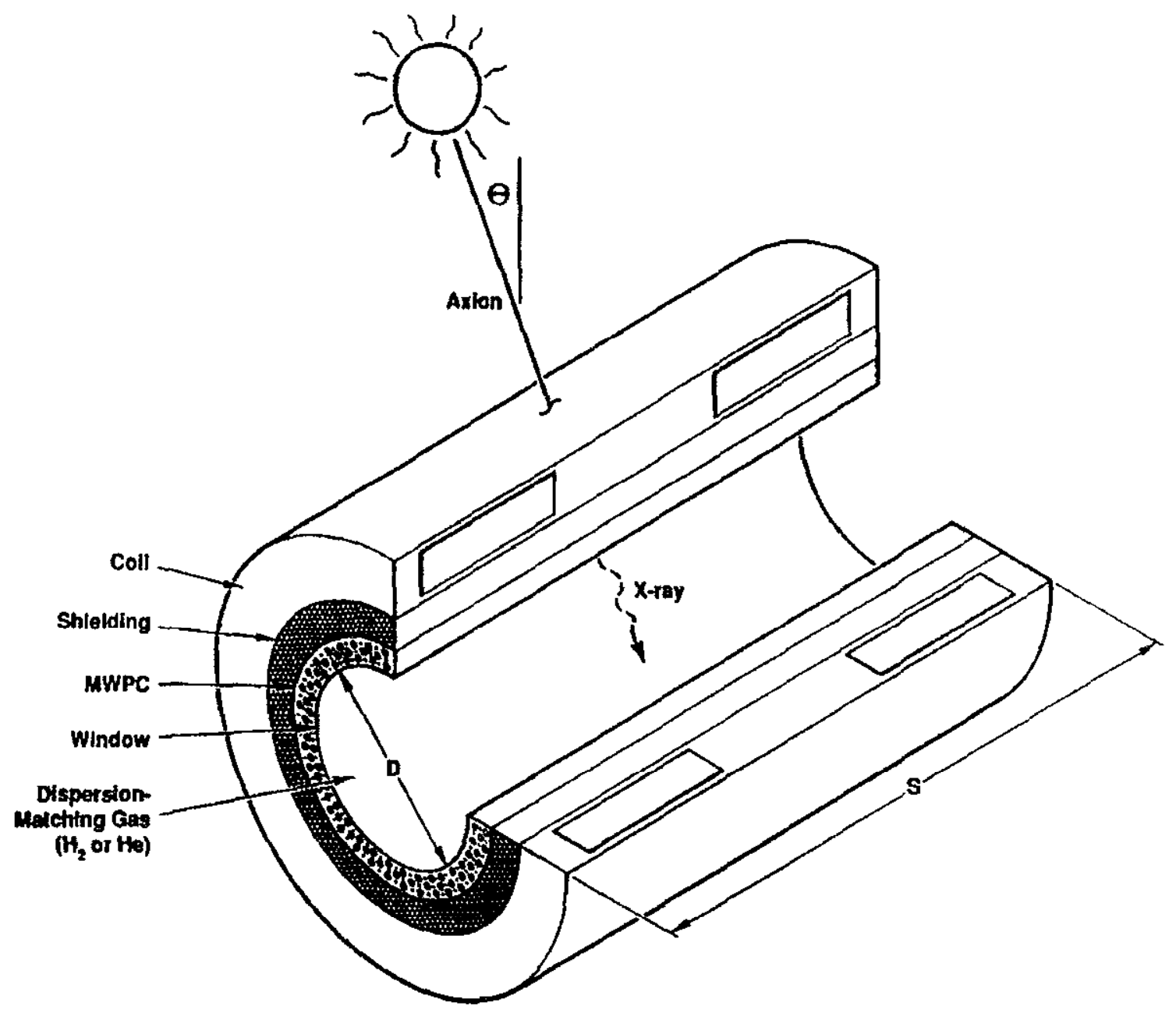

Figure 23 


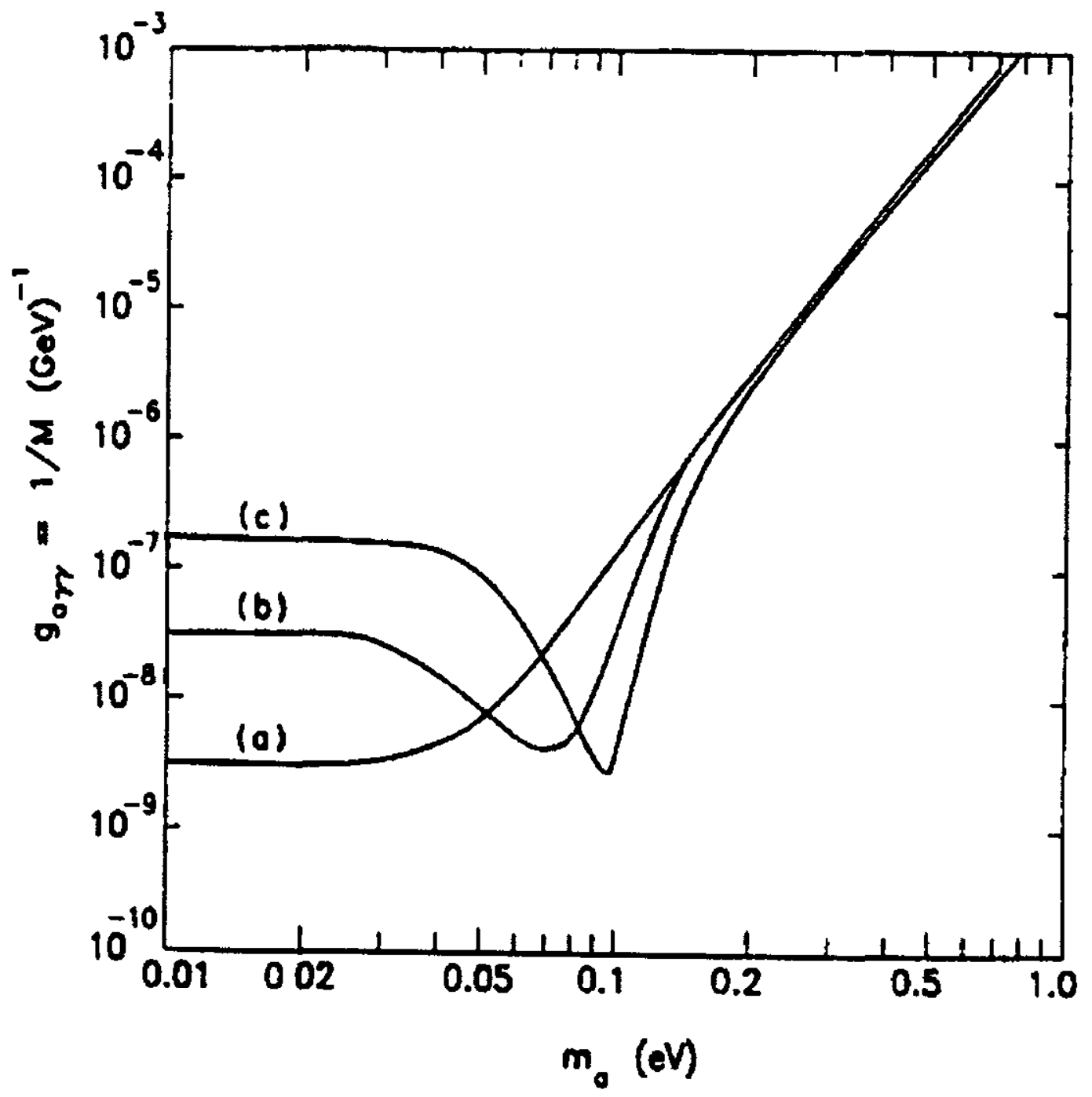

Figure 24 


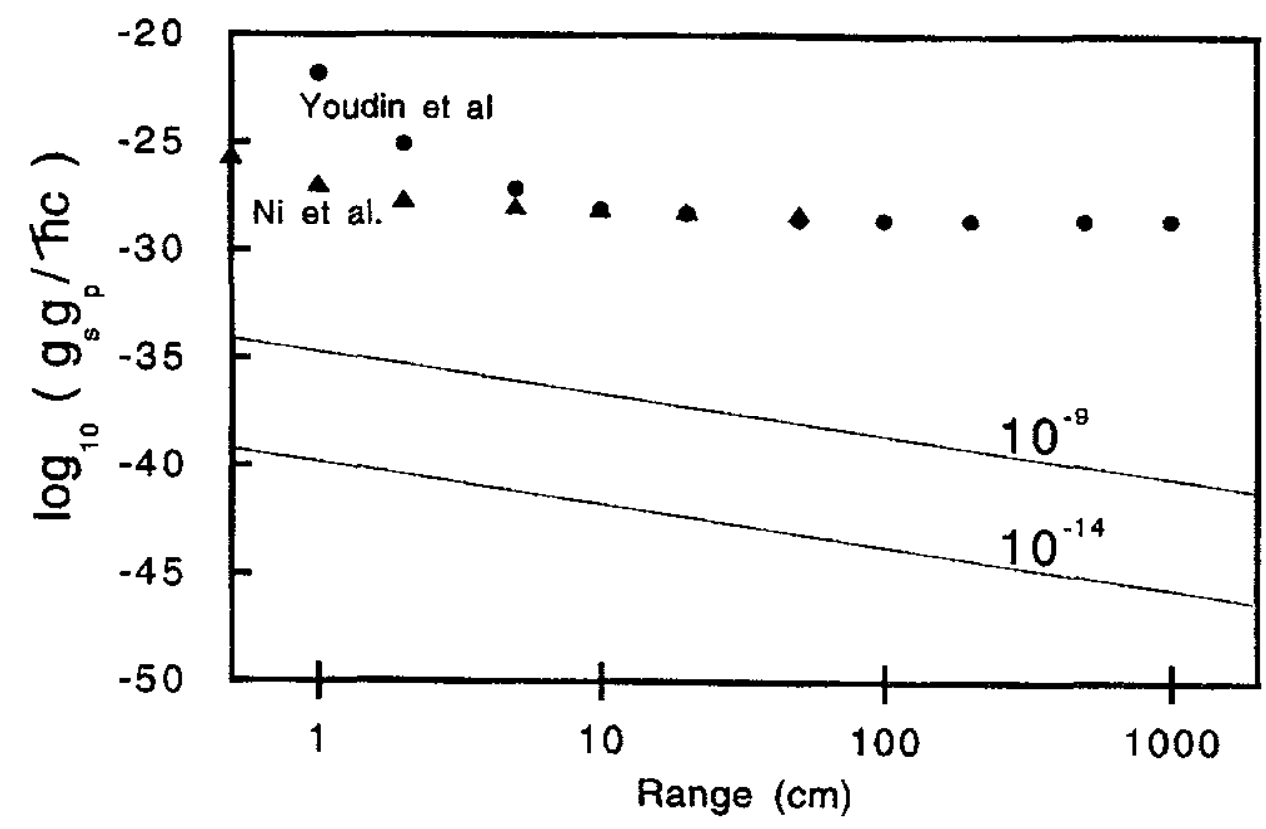

Figure 25

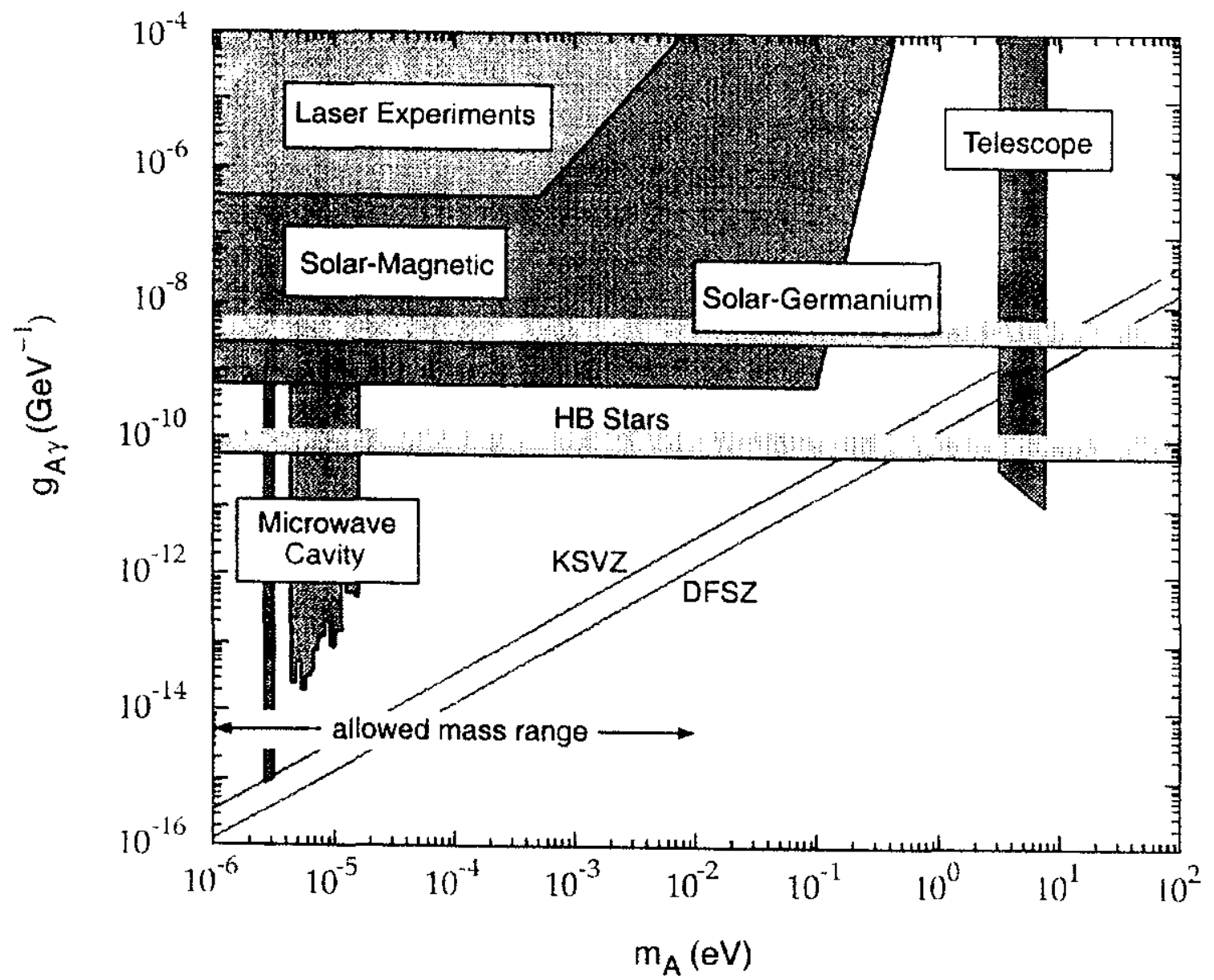

Figure 26 


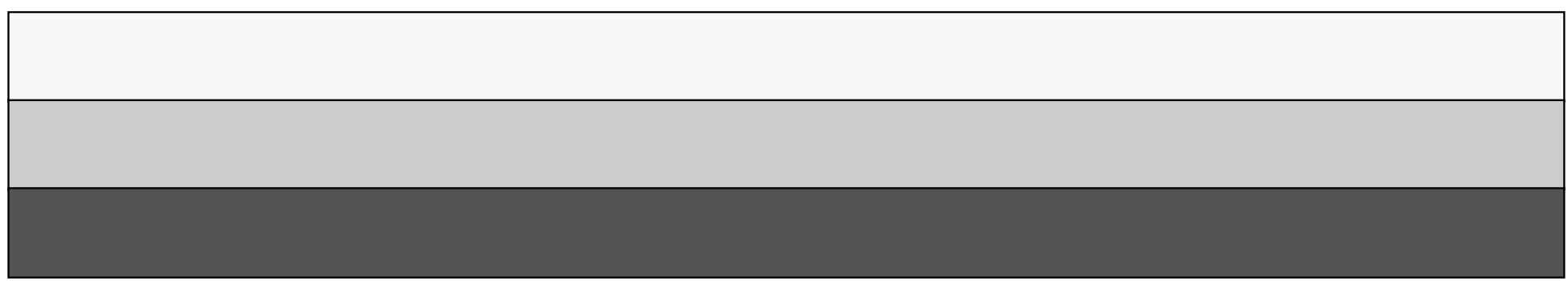

